\title{
Exactly thermalized quantum dynamics of the spin-boson model coupled to a dissipative environment
}

\author{
M. A. Lane $\odot,{ }^{1}$ D. Matos $\odot,{ }^{1}$ I. J. Ford, ${ }^{2}$ and L. Kantorovich ${ }^{1}$ \\ ${ }^{1}$ Department of Physics, King's College London, Strand, London WC2R 2LS, United Kingdom \\ ${ }^{2}$ Department of Physics and Astronomy, University College London, Gower Street, London WC1E 6BT, United Kingdom
}

(Received 19 February 2020; revised manuscript received 7 April 2020; accepted 7 April 2020; published 22 June 2020)

\begin{abstract}
We present an application of the extended stochastic Liouville-von Neumann equation (ESLN) method introduced earlier [G. M. G. McCaul, C. D. Lorenz, and L. Kantorovich, Phys. Rev. B 95, 125124 (2017); 97, 224310 (2018)], which describes the dynamics of an exactly thermalized open quantum system reduced density matrix coupled to a non-Markovian harmonic environment. Critically, the combined system of the open system fully coupled to its environment is thermalized at finite temperature using an imaginary-time evolution procedure before the application of real-time evolution. This initializes the combined system in the correct canonical equilibrium state rather than being initially decoupled. Here we apply our theory to the spin-boson Hamiltonian and develop a number of competing ESLN variants designed to reduce the numerical divergence of the trace of the open-system density matrix. We find that a careful choice of the driving noises is essential for improving numerical stability. We also investigate the effect of applying higher-order numerical schemes for solving stochastic differential equations, such as the Stratonovich-Heun scheme, and conclude that stochastic sampling dominates convergence with the improvement associated with the numerical scheme being less important for short times but required for late times. To verify the method and its numerical implementation, we first consider evolution under a fixed Hamiltonian and show that the system either remains in, or approaches, the correct canonical equilibrium state at long times. Additionally, evolution of the open system under nonequilibrium Landau-Zener (LZ) driving is considered and the asymptotic convergence to the LZ limit was observed for vanishing system-environment coupling and temperature. When coupling and temperature are nonzero, initially thermalizing the combined system at a finite time in the past was found to be a better approximation of the true LZ initial state than starting in a pure state.
\end{abstract}

DOI: 10.1103/PhysRevB.101.224306

\section{INTRODUCTION}

In open quantum systems, interactions between the system of interest and its environment drive behaviors which are not found in isolation such as dissipation and decoherence. Such phenomena play a strong role in quantum computing [1] and quantum thermodynamics [2] where the ability of an open system to stay in a superposition of states is desirable, though the treatment of such systems is analytically and numerically challenging. Existing methods are typically characterized by use of the reduced density matrix, obtained by taking the partial trace over the environment variables of the full density matrix. This began with the development of the Feynman-Vernon influence functional formalism where the response of a linear bath is expressed as a path integral over an infinite number of displaced harmonic oscillators [3]. Several techniques have since been developed, including hierarchical equations of motion [4,5], stochastic Liouville-von Neumann equations [6-10], stochastic Schrödinger equations [11], and quasiadiabatic path integrals [12]. Importantly, none of these methods make the Markov assumption, where environment correlation times are taken to be negligibly short compared to the characteristic timescales of the system of interest. This assumption has the physical interpretation that any information dissipated from the system to the environment will never be returned; i.e., the system-environment coupling is memoryless. However, these methods do assume that the system of interest and its environment are initially partitioned from each other; that is, they are initially decoupled and thermalized independently rather than as one combined system. This is fundamentally unphysical, especially for driven systems where a partitioned state is certainly not a good approximation of the correct initial thermal state and leads to incorrect transient dynamics with the possibility of the wrong asymptotic behavior.

This is not the case for the recently proposed extended stochastic Liouville-von Neumann equation (ESLN) method [10], which builds on the earlier work of Graber, Schramm, and Ingold [13] and allows one to derive the equations of motion for the reduced density matrix of an open quantum system without assuming a partitioned initial state. The theory considers a formally exact solution of the Liouville equation for the density matrix of the combined system, consisting of the open system and a harmonic (bosonic) environment (bath). Using the path-integral method, one can calculate exactly the reduced density matrix for the open system by integrating out the bath degrees of freedom. The resulting representation for the open system reduced density matrix contains nonlocal 
path integrals that can only be calculated numerically with considerable effort, assuming a finite number of time divisions (and hence integrals). Instead, we apply the HubbardStratonovich transformation to recast the density matrix as an average of a stochastic density matrix which satisfies simple stochastic differential equations with colored Gaussian noises. These noises are constrained to satisfy specific correlation functions which are fixed by the environment and its interaction with the open system (including the coupling strength), and which explicitly contain the temperature.

The theory provides an exact, nonperturbative description of the dynamics of an open system in the harmonic bath. There are two stochastic differential equations (SDEs): one in imaginary time that thermalizes the coupled system and the environment as a whole, and a second being the typical stochastic Liouville-von Neumann equation (SLN) for the open system. The thermalized state obtained at the end of an imaginary-time evolution becomes the initial state for the SLN so that sampling over all manifestations of the noises leads to the exact dynamics of the reduced density matrix of the open system starting in its thermal state. Equations for observables can then be obtained in the usual way. Crucially, the real-time SLN dynamics is affected by the coupling of the system to the environment during thermal preparation through the correlation between the real-time and imaginary-time noises. This has the natural interpretation that the preparation of the system may influence any early-time transient dynamics and perhaps even its asymptotic behavior at long times.

To simulate these stochastic differential equations, particular care should be taken with the choice of numerical scheme and the manner by which the colored noises are generated. The latter point is not trivial as the correlation functions in real time, imaginary time, and a cross-time correlation between them must be satisfied with sensible choices being made [14]. It turns out that some allowed choices result in numerical instability during the early-time dynamics, even though the correlation functions are fully satisfied. In our previous work [15], a method for noise generation was proposed which we shall review and further develop here, introducing a modified noise generation scheme that diminishes the exponential growth of the trace of the density matrix which seems to characterize these methods. This is the latest in a series of proposals aimed at tackling this problem $[16,17]$.

To test the accuracy of the ESLN method, the spin-boson model will be considered as the test bed. It is typically the initial starting model for any approach that deals with open quantum systems, due to its relative simplicity while still exhibiting dissipative behavior. The model consists of a twolevel spin system surrounded by bosonic degrees of freedom that describe the environment, and can naturally be applied to qubits coupled to an environment [18-22], electronic energy transfer in biological systems [16], Josephson junctions [23-25], cold atoms [26,27], and solid-state artificial atoms [28]. The spin-boson model has already been considered previously by us in the context of the ESLN [15]; however, due to a recently discovered implementation error, the numerical results were inaccurate. Here we present further implementation development and update our numerical results.

So, the purpose of the present paper is fourfold: (i) review and extend existing methods of solving the dynamics of open quantum systems when the density matrix is initialized in the correct canonical equilibrium state; (ii) pay special attention to the generation of colored Gaussian noises for both real- and imaginary-time evolutions; (iii) examine the convergence properties of two numerical schemes, one of which uses Stratonovich calculus; and (iv) test the numerical behavior of different trace-preserving forms of the ESLN and explain their divergent behavior in detail. In Sec. II we briefly review the ESLN [10] before moving on to the spin-boson model. Section III presents the schemes for noise generation along with techniques for reducing the exponential growth of the trace, while Sec. IV discusses various forms of the ESLN including two trace-preserving forms obtained via a Girsanov transformation $[9,29]$. In Sec. V we discuss schemes for solving the ESLN numerically using methods rooted in stochastic calculus. Results of numerical simulations are given in Sec. VI and the discussion and conclusions are presented in Sec. VII.

\section{THEORY}

\section{A. Extended stochastic Liouville-von Neumann equations}

Following the influence functional formalism of Feynman and Vernon [3], we consider the standard setup of an open quantum system with coordinates $q$ and Hamiltonian $H_{q}$ that may describe either an electronic or bosonic subsystem, or both, and may depend explicitly on time. This system is coupled to its environment: a heat bath of harmonic atoms $i$ with masses $m_{i}$, and a potential energy that is quadratic in their displacement coordinates $\xi_{i}$.

The coupling between the open system and its environment is linear in the environment coordinates but fully general in $q$, taking the form $-\xi_{i} f_{i}(q)$, with the set of $f_{i}(q)$ being arbitrary functions of $q$. The full system Hamiltonian is thus

$$
\begin{aligned}
H_{\mathrm{tot}}\left(q,\left\{\xi_{i}\right\}, t\right)= & H_{q}(q, t)+\sum_{i} \frac{p_{i}^{2}}{2 m_{i}}+\frac{1}{2} \sum_{i j} \Lambda_{i j} \xi_{i} \xi_{j} \\
& -\sum_{i} \xi_{i} f_{i}(q)
\end{aligned}
$$

where $p_{i}$ are momenta coordinates canonical to $\xi_{i}$, and $\Lambda_{i j}$ is the force constant matrix of the bath. A transformation to normal modes then represents the bath as a set of noninteracting harmonic oscillators. This is a more general form of the Caldeira-Leggett Hamiltonian [30] since the environment coupling is a general function of $q$ rather than being strictly bilinear.

In typical studies, the open system and environment density matrix is initialized in a partitioned state where the full system density matrix $\rho_{0}=\rho_{\text {tot }}\left(t_{0}\right)$ is the tensor product of the open system density matrix $\boldsymbol{\rho}_{q}\left(t_{0}\right)$ and that of its environment $\boldsymbol{\rho}_{\xi}\left(t_{0}\right)$ at some initial time $t_{0}$,

$$
\boldsymbol{\rho}_{0}=\boldsymbol{\rho}_{q}\left(t_{0}\right) \otimes \boldsymbol{\rho}_{\xi}\left(t_{0}\right)
$$

The more appropriate and useful initial state would be the one where the open system and its environment are coupled and in thermal equilibrium. This can be obtained via appropriate 
preparation of the canonical equilibrium density matrix [13],

$$
\rho_{0}=\frac{1}{Z_{0}} e^{-\beta H_{0}},
$$

where $H_{0}=H_{\text {tot }}\left(t_{0}\right)$ is the initial Hamiltonian of the combined open system and its environment, $Z_{0}=\operatorname{Tr}\left(e^{-\beta H_{0}}\right)$ is the equilibrium partition function of the total system, and $\beta=1 / k_{B} T$ is the inverse temperature.

Following the seminal work of Graber, Schramm, and Ingold [13], it was recently shown $[10,15]$ that it is possible to thermalize the reduced density matrix of the open system via a novel application of the influence functional formalism in which the environment variables are integrated out for arbitrary real time $t$. The resulting pair of SDEs describing the thermalization in imaginary time and subsequent dynamics in real time of the stochastic reduced density matrix are known as the extended stochastic Liouville-von Neumann equations (ESLNs), with the evolution of the reduced density matrix being driven by complex correlated Gaussian noises in both cases. Expressing the equation of motion of the physical reduced density matrix as an ensemble average over stochastic paths via a Hubbard-Stratonovich transformation in this way is commonly referred to as stochastic unraveling [31-33].

Thermalization is described by the evolution in imaginary time $\tau$ of a density matrix $\bar{\rho}(\tau)$ over the domain $\tau \in[0, \beta \hbar]$ via

$$
-\hbar \frac{d \bar{\rho}(\tau)}{d \tau}=\left[H_{q}\left(t_{0}\right)+\sum_{i} \mu_{i}(\tau) f_{i}(q)\right] \bar{\rho}(\tau),
$$

with $\bar{\rho}(\tau)$ initialized in the unitary state, $\bar{\rho}(\tau=0)=\mathbb{I}$. The final value of this evolution at $\tau=\beta \hbar$ corresponds to the equilibrium density matrix, up to a normalization constant which will be fixed later. This is then used as the initial condition for the real-time dynamics of the reduced density matrix which satisfies

$$
\begin{aligned}
i \hbar \frac{d \rho(t)}{d t}= & {\left[H_{q}(t), \rho(t)\right]-\sum_{i}\left(\eta_{i}(t)\left[f_{i}(q), \rho(t)\right]\right.} \\
& \left.+\frac{\hbar}{2} v_{i}(t)\left\{f_{i}(q), \rho(t)\right\}\right),
\end{aligned}
$$

where the square (curly) brackets represent the standard (anti)commutators.

The functions $\eta_{i}(t), v_{i}(t)$, and $\mu_{i}(\tau)$ are the complex Gaussian driving noises, distributed via the multivariate Gaussian

$$
\begin{aligned}
\mathcal{W} & {\left[\left\{\mu_{i}\right\},\left\{\eta_{i}\right\},\left\{v_{i}\right\}\right] } \\
= & \mathcal{N} \exp \left\{-\frac{1}{2}\left[\int_{0}^{t} d t^{\prime} \int_{0}^{t} d t^{\prime \prime} z_{1}^{T}\left(t^{\prime}\right) \boldsymbol{\Sigma}^{11}\left(t^{\prime}-t^{\prime \prime}\right) z_{1}\left(t^{\prime \prime}\right)\right.\right. \\
& +2 \int_{0}^{t} d t^{\prime} \int_{0}^{\beta \hbar} d \tau z_{1}^{T}\left(t^{\prime}\right) \boldsymbol{\Sigma}^{12}\left(t^{\prime}, \tau\right) z_{2}(\tau) \\
& \left.\left.+\int_{0}^{\beta \hbar} d \tau \int_{0}^{\beta \hbar} d \tau^{\prime} z_{2}^{T}\left(\tau^{\prime}\right) \Sigma^{22}\left(\tau-\tau^{\prime}\right) z_{2}\left(\tau^{\prime}\right)\right]\right\}
\end{aligned}
$$

and arising from the application of a two-time HubbardStratonovich transformation $[8,10,34]$ to the environment influence functional. Here $\mathcal{N}$ is a normalization constant, $z_{1}=$ $\left(\left\{\eta_{i}\right\}\left\{\eta_{i}^{*}\right\}\left\{v_{i}\right\}\left\{v_{i}^{*}\right\}\right)^{T}$ and $z_{2}=\left(\left\{\mu_{i}\right\}\left\{\mu_{i}^{*}\right\}\right)^{T}$ are the vector noises, and the $\Sigma^{i j}$ are time-dependent matrices to be discussed shortly.

The physical reduced density matrix is obtained by the average $\langle\ldots\rangle$ of an ensemble of stochastic reduced density matrices, taken over the noises with the multivariate Gaussian weighting given above. In particular, the average at the end of imaginary-time evolution yields the exact thermalized initial state of the real-time evolution, that is, $\boldsymbol{\rho}^{p h}\left(t_{0}\right)=\mathbb{N}\langle\overline{\boldsymbol{\rho}}(\beta \hbar)\rangle \equiv$ $\mathbb{N}\left\langle\rho\left(t_{0}\right)\right\rangle$. Here, $\mathbb{N}$ is a time-independent prefactor that is to be fixed $[10,15]$ after sampling using the condition $\operatorname{Tr}\left[\rho^{p h}(t)\right]=$ $\mathbb{N} \operatorname{Tr}[\langle\rho(t)\rangle]=1$. In practice this can be done at any time including $t_{0}$, so the physical density matrix is obtained by taking $\mathbb{N}=1 / \operatorname{Tr}\left[\left\langle\rho\left(t_{0}\right)\right\rangle\right]$.

The blocks of the matrix $\Sigma=\left(\begin{array}{ll}\Sigma^{11} & \Sigma^{12} \\ \Sigma^{22}\end{array}\right)$ in the Gaussian of Eq. (6) are defined such that the corresponding elements of its inverse are equal to the appropriate noise correlation functions (given below). Only correlation functions between noises $\eta_{i}(t), v_{i}(t)$, and $\mu_{i}(\tau)$ are needed; other correlation functions involving complex conjugated noises can be ignored [15]. It is important to note that each realization of these noises will produce a unique trajectory describing an initial thermalized stochastic density matrix and its subsequent realtime dynamics, with the physical density matrix obtained by stochastic averaging over a sufficiently large sample of such realizations. This has the pleasingly intuitive interpretation of averaging over all possible behaviors of the bath, reminiscent of the direct link to the sum over all possible paths in the path-integral representation, only now this sum is replaced by the stochastic average over environmental noises.

We emphasize that this not an ad hoc representation of the system where the stochastic fields would have been introduced artificially to model the environment. Instead, they have been introduced and their properties derived rigorously from an appropriate consideration of the whole system, consisting of both the open system and its bosonic environment, by means of elimination of the environment using the path-integral method and the Hubbard-Stratonovich transformation.

\section{B. Noise correlation functions}

The noises are defined by their site-dependent correlation functions,

$$
\begin{gathered}
\left\langle\eta_{i}(t) \eta_{j}\left(t^{\prime}\right)\right\rangle=\frac{\hbar}{\sqrt{m_{i} m_{j}}} \sum_{\lambda} \frac{e_{\lambda i} e_{\lambda j}}{2 \omega_{\lambda}} \operatorname{coth}\left(\frac{1}{2} \beta \hbar \omega_{\lambda}\right) \cos \left(\omega_{\lambda} t\right), \\
\left\langle\eta_{i}(t) v_{j}\left(t^{\prime}\right)\right\rangle=-\frac{2 i \Theta\left(t-t^{\prime}\right)}{\sqrt{m_{i} m_{j}}} \sum_{\lambda} \frac{e_{\lambda i} e_{\lambda j}}{2 \omega_{\lambda}} \operatorname{coth}\left(\frac{1}{2} \beta \hbar \omega_{\lambda}\right) \\
\times \sin \left(\omega_{\lambda} t\right), \\
\left\langle\eta_{i}(t) \mu_{j}(\tau)\right\rangle \\
=-\frac{\hbar}{\sqrt{m_{i} m_{j}}} \sum_{\lambda} \frac{e_{\lambda i} e_{\lambda j}}{2 \omega_{\lambda}} \frac{\cosh \left[\frac{1}{2} \beta \hbar \omega_{\lambda}-i \omega_{\lambda}(t-i \tau)\right]}{\sinh \left(\frac{1}{2} \beta \hbar \omega_{\lambda}\right)}
\end{gathered}
$$




$$
\begin{gathered}
\left\langle\mu_{i}(\tau) \mu_{j}\left(\tau^{\prime}\right)\right\rangle=\frac{\hbar}{\sqrt{m_{i} m_{j}}} \sum_{\lambda} \frac{e_{\lambda i} e_{\lambda j}}{2 \omega_{\lambda}}\left[\operatorname{coth}\left(\frac{1}{2} \beta \hbar \omega_{\lambda}\right)\right. \\
\left.\times \cosh \left(\omega_{\lambda} \tau\right)-\sinh \left(\omega_{\lambda} \tau\right)\right] \\
\left\langle v_{i}(t) v_{j}\left(t^{\prime}\right)\right\rangle=\left\langle v_{i}(t) \mu_{j}(\tau)\right\rangle=0
\end{gathered}
$$

where $\Theta(t)$ is the Heaviside step function. Here, the $e_{\lambda}$ are the eigenvectors of the bath dynamical matrix, $D_{i j}=$ $\Lambda_{i j} / \sqrt{m_{i} m_{j}}$, with eigenvalues $\omega_{\lambda}^{2}$.

In the standard SLN without any thermalization, there would be no $\mu$ noise and no $\eta-\mu$ correlation. This is indicative of the neglected information inherent in initializing the system in a partitioned state. In the thermalized ESLN, thermalization leads to entanglement between the system of interest and its environment, manifested in the $\eta-\mu$ cross correlation, which may persist after thermalization during the real-time dynamics. At first glance this may seem strange, since the cross-correlation between real and imaginary times refers to two intrinsically different time coordinates. Regardless, the noises are auxiliary variables introduced by the application of a two-time Hubbard-Stratonovich transformation; they do not have physical meaning by themselves. Similarly, components of the stochastic density matrix are simply mathematical degrees of freedom from the perspective of the correlations functions, describing a random trajectory first along the imaginary coordinate $\tau$ and second along the real coordinate $t$, with the particular realization of the latter depending on the final realization of the former. The physical density matrix is obtained after averaging over these realizations, with each realization being a different stochastic quantum trajectory.

The general ESLN, Eqs. (4) and (5), requires three noises $\eta_{i}, v_{i}$, and $\mu_{i}$ per lattice site $i$. In normal mode representation $\lambda$ the correlation matrices are diagonalized. Next, by assuming that the system variable dependence of the systemenvironment coupling, $-\sum_{\lambda} f_{\lambda}(q) \xi_{\lambda}$, is the same for each mode up to a scaling factor, $f_{\lambda}(q)=c_{\lambda} f(q)$, the set of noise terms can be reduced from three per site down to only three [15]. For example, taking the $\eta_{i} \rightarrow \eta_{\lambda}$ noise, the $\eta_{i}$ term in Eq. (5) becomes

$$
\sum_{i} \eta_{i}(t)\left[f_{i}(q), \rho(t)\right] \rightarrow \eta(t)[f(q), \rho(t)]
$$

with $\eta(t)=\sum_{\lambda} c_{\lambda} \eta_{\lambda}(t)$ being a new Gaussian noise. The $\eta-\eta$ correlation function is then

$$
\left\langle\eta(t) \eta\left(t^{\prime}\right)\right\rangle=\hbar \sum_{\lambda} \frac{c_{\lambda}^{2}}{2 \omega_{\lambda}} \operatorname{coth}\left(\frac{1}{2} \beta \hbar \omega_{\lambda}\right) \cos \left[\omega_{\lambda}\left(t-t^{\prime}\right)\right]
$$

where the sum over environmental modes can be replaced by an integration over frequency in the continuum limit,

$$
\begin{aligned}
& \sum_{\lambda} \frac{c_{\lambda}^{2}}{2 \omega_{\lambda}} \cdots \rightarrow \int_{0}^{\infty} \frac{d \omega}{\pi}\left[\pi \sum_{\lambda} \frac{c_{\lambda}^{2}}{2 \omega_{\lambda}} \delta\left(\omega-\omega_{\lambda}\right)\right] \cdots \\
& \quad=\int_{0}^{\infty} \frac{d \omega}{\pi} J(\omega) \cdots .
\end{aligned}
$$

Here, $J(\omega)$ is the spectral density of the environment, taken in this work to be the Drude spectral density,

$$
J(\omega)=\alpha \omega\left[1+\left(\frac{\omega}{\omega_{c}}\right)^{2}\right]^{-2}
$$

where $\alpha$ is proportional to the squares of the $c_{\lambda}$ coefficients and so parametrizes the effective coupling strength between the system and environment. $\omega_{c}$ is the Drude-Lorentz cutoff frequency which ensures that the density goes smoothly to zero as $\omega$ becomes large [22,35].

Just as for the $\eta$ noise, the sets of $\nu_{i}$ and $\mu_{i}$ noises may be reduced to only a single $v$ and $\mu$ Gaussian noise, and the sums over $i$ in Eqs. (4) and (5) are completely removed. The correlation functions for these three reduced noises are

$$
\begin{aligned}
\left\langle\eta(t) \eta\left(t^{\prime}\right)\right\rangle= & \hbar \int_{0}^{\infty} \frac{d \omega}{\pi} J(\omega) \operatorname{coth}\left(\frac{1}{2} \beta \hbar \omega\right) \cos \left[\omega\left(t-t^{\prime}\right)\right] \\
\equiv & K_{\eta \eta}\left(t-t^{\prime}\right), \\
\left\langle\eta(t) \nu\left(t^{\prime}\right)\right\rangle= & -2 i \Theta\left(t-t^{\prime}\right) \int_{0}^{\infty} \frac{d \omega}{\pi} J(\omega) \sin \left[\omega\left(t-t^{\prime}\right)\right] \\
\equiv & K_{\eta \nu}\left(t-t^{\prime}\right), \\
\langle\eta(t) \mu(\tau)\rangle= & -\hbar \int_{0}^{\infty} \frac{d \omega}{\pi} J(\omega) \frac{\cosh \left[\frac{1}{2} \beta \hbar \omega-i \omega(t-i \tau)\right]}{\sinh \left(\frac{1}{2} \beta \hbar \omega\right)} \\
\equiv & K_{\eta \mu}(t, \tau), \\
\left\langle\mu(\tau) \mu\left(\tau^{\prime}\right)\right\rangle= & \hbar \int_{0}^{\infty} \frac{d \omega}{\pi} J(\omega)\left[\cosh \left[\omega\left(\tau-\tau^{\prime}\right)\right]\right. \\
& \left.\times \operatorname{coth}\left(\frac{1}{2} \beta \hbar \omega\right)-\sinh \left[\omega\left(\tau-\tau^{\prime}\right)\right]\right] \\
\equiv & K_{\mu \mu}\left(\tau-\tau^{\prime}\right), \\
\left\langle\nu(t) v\left(t^{\prime}\right)\right\rangle= & \langle v(t) \mu(\tau)\rangle=0, \quad \forall t, t^{\prime}, \tau,
\end{aligned}
$$

where we have defined so-called physical kernels on the righthand sides. Note that Eq. (19) is possible because the noises are complex valued. Correspondingly, Eqs. (4) and (5) are simplified as

$$
\begin{aligned}
-\hbar \frac{d \bar{\rho}(\tau)}{d \tau}= & {\left[H_{q}\left(t_{0}\right)+\mu(\tau) f(q)\right] \bar{\rho}(\tau), } \\
i \hbar \frac{d \rho(t)}{d t}= & {\left[H_{q}(t), \rho(t)\right]-\eta(t)[f(q), \rho(t)] } \\
& -\frac{\hbar}{2} v(t)\{f(q), \rho(t)\} .
\end{aligned}
$$

Note that formally Eq. (21) coincides with the SLN dynamics. The important difference here lies in the cross-correlation with the imaginary-time dynamics associated with thermalization, and the use of the final result of each $\bar{\rho}(\tau)$ as the initial condition for each $\rho(t)$.

\section{Spin-boson model}

Thus far, the system Hamiltonian $H_{q}$ has been kept fully general, as has the form of the system-environment coupling, $f(q)$. The spin-boson Hamiltonian for a generic two-state 
system,

$$
\begin{aligned}
H_{q}(t) & =\frac{1}{2} \hbar \Delta(t) \sigma_{x}+\frac{1}{2} \hbar \epsilon(t) \sigma_{z} \\
& =\frac{1}{2} \hbar \Delta(t)(|0\rangle\langle 1|+| 1\rangle\langle 0|)+\frac{1}{2} \hbar \epsilon(t)(|0\rangle\langle 0|-| 1\rangle\langle 1|),
\end{aligned}
$$

is a good model in which to confirm the efficacy of the ESLN. Here $\sigma_{x}$ and $\sigma_{z}$ are the standard Pauli spin matrices with $\sigma_{x}$ flipping the spin from one state to the other with tunneling strength $\Delta(t)$, and $\sigma_{z}$ biasing the states with magnitude $\epsilon(t)$. The system-bath coupling is just $\sigma_{z}$ so that Eqs. (20) and (21) become

$$
\begin{aligned}
-\hbar \frac{d \bar{\rho}(\tau)}{d \tau} & =\left[H\left(t_{0}\right)+\mu(\tau) \sigma_{z}\right] \bar{\rho}(\tau), \\
i \hbar \frac{d \rho(t)}{d t} & =[H(t), \rho(t)]-\eta(t)\left[\sigma_{z}, \rho(t)\right]-\frac{\hbar}{2} v(t)\left\{\sigma_{z}, \rho(t)\right\} .
\end{aligned}
$$

The total system is first jointly thermalized using Eq. (23) so that at $\tau=\beta \hbar$ the sample average produces the equilibrium state Eq. (3). Each stochastic $\rho(t)$ is then initialized at $t_{0}$ in the corresponding equilibrium state $\bar{\rho}(\beta \hbar)$ and evolved in real time according to Eq. (24). Finally, the normalization factor $\mathbb{N}$ is determined and the full physical reduced density matrix becomes completely defined.

In this work two simple tests for the dynamics are discussed. First, we consider equilibrium evolution with constant driving whereby the system decays toward the thermal state if initialized elsewhere or remains unperturbed if initialized in the thermal state. And second, a linear driving after some initial time $t_{0}$ of the form $\epsilon(t)=\kappa t$ with constant $\Delta$ is investigated, known as the Landau-Zener sweep [36]. Importantly, for an isolated spin being linearly driven from $\epsilon(-\infty)=-\infty$ to $\epsilon(+\infty)=\infty$ at zero temperature starting in the ground state $|1\rangle$, or $\rho_{i j}(-\infty)=\delta_{i 1} \delta_{j 1}$, the survival probability as $t \rightarrow \infty$ is $[11,36-40]$

$$
P_{L Z}=\exp \left\{-\frac{\pi \Delta^{2}}{2 \hbar \kappa}\right\}
$$

which corresponds to an asymptotic mean $z$ spin of

$$
\left\langle\sigma_{z}\right\rangle_{L Z}=2 \exp \left\{-\frac{\pi \Delta^{2}}{2 \hbar \kappa}\right\}-1 .
$$

Though this result was originally derived for an isolated spin, it has since been shown that the same asymptotic behavior is valid for a dissipative spin coupled to a harmonic environment at zero temperature, where coupling is provided entirely via $\sigma_{z}[11,38,39,41]$. This correspondence breaks down if the initial condition is not the ground state $|1\rangle$ in the infinite past, or for nonzero temperature.

Finally, using Eqs. (23) and (24) for the spin-boson Hamiltonian it is straightforward to derive coupled SDEs for the $x, y$, and $z$ spins and also for the $\operatorname{trace}, \operatorname{Tr}[\rho(t)]$,

$$
\begin{aligned}
& \hbar \frac{d \sigma_{x}(t)}{d t}=-[\epsilon(t)-2 \eta(t)] \sigma_{y}(t), \\
& \hbar \frac{d \sigma_{y}(t)}{d t}=-\Delta \sigma_{z}(t)+[\epsilon(t)-2 \eta(t)] \sigma_{x}(t),
\end{aligned}
$$

$$
\begin{aligned}
\hbar \frac{d \sigma_{z}(t)}{d t} & =\Delta \sigma_{y}(t)+i v(t) \operatorname{Tr}[\rho(t)], \\
\hbar \frac{d \operatorname{Tr}[\rho(t)]}{d t} & =i v(t) \sigma_{z}(t),
\end{aligned}
$$

where the last equation is obtained by taking the trace of Eq. (24). To be clear, here $\sigma_{i}$ without time is just the usual Pauli spin matrix, while $\sigma_{i}(t)=\operatorname{Tr}\left[\sigma_{i} \rho(t)\right]$ is the quantum average using a single realization of the density matrix, and

$$
\left\langle\sigma_{i}(t)\right\rangle=\operatorname{Tr}\left[\sigma_{i} \boldsymbol{\rho}^{p h}(t)\right]=\operatorname{Tr}\left(\sigma_{i} \frac{\langle\boldsymbol{\rho}(t)\rangle}{\operatorname{Tr}\left[\left\langle\boldsymbol{\rho}\left(t_{0}\right)\right\rangle\right]}\right)
$$

is the quantum average using the physical density matrix obtained after stochastic averaging and normalization.

\section{NOISES}

\section{A. Noise generation scheme}

Compared to the SLN, the noises in the ESLN have the additional complexity of an extra colored noise $\mu$ with its own time coordinate $\tau$, introducing cross-time correlations [10]. Adopting the notation for the noises used in [15], the correlation functions for the spin-boson Hamiltonian reduce to Eqs. (15)-(19). These correlation functions act as constraints on any noise generated, but the noises are not uniquely defined by them. This provides some freedom in specifying the generation procedure, as long as the correlation functions are satisfied.

Decomposing each noise into its orthogonal components such that each component is correlated with only one other component, and denoting the correlations between components with subscripts, the noises can be written as

$$
\begin{aligned}
\eta(t) & =\eta_{\eta}(t)+\eta_{\nu}(t)+\eta_{\mu}(t), \\
\nu(t) & =v_{\eta}(t), \\
\mu(\tau) & =\mu_{\mu}(\tau)+\mu_{\eta}(\tau) .
\end{aligned}
$$

Explicitly, this means that $\eta_{v}$ is only correlated with $v_{\eta}$, with equivalent products for other orthogonal pairs. This orthogonality can be achieved by expressing each component as a convolution of an unknown time function $G$ (to be called a filtering kernel) with a sum of real-valued white noises, satisfying

$$
\begin{gathered}
\left\langle x_{i}(t) x_{j}\left(t^{\prime}\right)\right\rangle=\delta_{i j} \delta\left(t-t^{\prime}\right), \\
\left\langle\bar{x}_{i}(\tau) \bar{x}_{j}\left(\tau^{\prime}\right)\right\rangle=\delta_{i j} \delta\left(\tau-\tau^{\prime}\right), \\
\left\langle x_{i}(t) \bar{x}_{j}(\tau)\right\rangle=0 \text { for } \forall i, j .
\end{gathered}
$$

Here $x_{i}(t)$ and $\bar{x}_{i}(\tau)$ refer to a white noise in real and imaginary time, respectively. The convolutions thus take the form

$$
\begin{aligned}
& \eta_{\eta}(t)=\int_{-\infty}^{\infty} d t^{\prime} G_{\eta \eta}\left(t-t^{\prime}\right) x_{1}\left(t^{\prime}\right), \\
& \eta_{\nu}(t)=\int_{-\infty}^{\infty} d t^{\prime} G_{\eta \nu}\left(t-t^{\prime}\right)\left[x_{2}\left(t^{\prime}\right)+i x_{3}\left(t^{\prime}\right)\right], \\
& \eta_{\mu}(t)=\int_{0}^{\beta \hbar} d \tau G_{\eta \mu}(t, \tau)\left[\bar{x}_{2}(\tau)+i \bar{x}_{3}(\tau)\right],
\end{aligned}
$$




$$
\begin{aligned}
v_{\eta}(t) & =\int_{-\infty}^{\infty} d t^{\prime} G_{\nu \eta}\left(t-t^{\prime}\right)\left[x_{3}\left(t^{\prime}\right)+i x_{2}\left(t^{\prime}\right)\right], \\
\mu_{\mu}(\tau) & =\int_{-\beta \hbar}^{\beta \hbar} d \tau^{\prime} G_{\mu \mu}\left(\tau-\tau^{\prime}\right) \bar{x}_{1}\left(\tau^{\prime}\right), \\
\mu_{\eta}(\tau) & =\int_{0}^{\beta \hbar} d \tau^{\prime} G_{\mu \eta}\left(\tau-\tau^{\prime}\right)\left[\bar{x}_{3}\left(\tau^{\prime}\right)+i \bar{x}_{2}\left(\tau^{\prime}\right)\right],
\end{aligned}
$$

from which it is straightforward to show that the expectation values of component pairs correspond to the appropriate correlation functions, e.g., $\left\langle\eta(t) v\left(t^{\prime}\right)\right\rangle=\left\langle\eta_{v}(t) v_{\eta}\left(t^{\prime}\right)\right\rangle$. The choice of each $G$ is made by equating the expectation values of the noises to the appropriate physical kernels, $K(t)$, Eqs. (15)(19), and taking Fourier transforms (indicated by the tilde) where appropriate to obtain

$$
\begin{aligned}
\tilde{G}_{\eta \eta}(\omega) & =\sqrt{\tilde{K}_{\eta \eta}(\omega)}, \\
\tilde{G}_{\eta \nu}(\omega) & =\tilde{G}_{\nu \eta}(-\omega)=\sqrt{-\frac{i}{2} \tilde{K}_{\eta \nu}(\omega),} \\
\tilde{G}_{\mu \mu}(\omega) & =\sqrt{\tilde{K}_{\mu \mu}(\omega)}, \\
G_{\eta \mu}(t, \tau) & =-\frac{i}{2} K_{\eta \mu}(t-i \tau),
\end{aligned}
$$

with the remaining filtering kernel given by a delta function $G_{\mu \eta}(\tau)=\delta(\tau)$. Note that in our previous work [15] we used $G_{\nu \eta}(t)=\delta(t)$ instead of Eq. (45), which we have found leads to much less stable dynamics [14]. The noises can then be obtained by applying the convolution theorem to Eqs. (38)(42) before taking the inverse Fourier transform.

\section{B. Variance reduction technique}

From the equation of motion for the trace, Eq. (30), and given that $v$ is complex valued, it is found that the trace can grow exponentially in time [17], requiring punitively large sampling for convergence. Recent proposals to optimize the noise generation method $[16,17]$ have managed to reduce this growth by many orders of magnitude, though here we present a much simpler method of exploiting the relative magnitudes of correlated pairs of orthogonal noises such that their correlation functions do not change.

Since the noise components are orthogonal, the correlation functions depend only on the two appropriate components, e.g., $K_{\eta v}\left(t-t^{\prime}\right)=\left\langle\eta_{v}(t) v_{\eta}\left(t^{\prime}\right)\right\rangle$, so $v_{\eta}$ can be multiplied and $\eta_{\nu}$ divided by the same factor without modifying the correlation, and equivalently for $K_{\eta \mu}$. To accomplish this, we define the scaling factors

$$
\begin{aligned}
& a_{\mu \eta}=\sqrt{r_{\mu \eta}} \sqrt{\frac{\frac{1}{M} \sum_{m=0}^{M}\left|\mu_{\eta}\left(\tau_{m}\right)\right|}{\max _{n}\left|\eta_{\mu}\left(t_{n}\right)\right|}}, \\
& b_{\nu \eta}=\sqrt{r_{\nu \eta}} \sqrt{\frac{\sum_{n=0}^{N}\left|v_{\eta}\left(t_{n}\right)\right|}{\sum_{n=0}^{N}\left|\eta_{\nu}\left(t_{n}\right)\right|}},
\end{aligned}
$$

where $M=\beta \hbar / d \tau$ and $N=t_{\max } / d t$ are the number of real and imaginary time steps, respectively, with $\tau_{m}=m d \tau$ and $t_{n}=n d t$, while $r_{\mu \eta}$ and $r_{v \eta}$ are the desired average ratios of the relative components of the noises over a single realization. The desired new noises are thus obtained by simply rescaling the components as $\eta_{\mu}^{\text {new }}=a_{\mu \eta} \eta_{\mu}$ and $\mu_{\eta}^{\text {new }}=\mu_{\eta} / a_{\mu \eta}$, and $\eta_{\nu}^{\text {new }}=b_{\nu \eta} \eta_{\nu}$ and $v_{\eta}^{\text {new }}=v_{\eta} / b_{\nu \eta}$. Here, the maximum absolute value of $\eta_{\mu}$ rather than the average over its realization is used in Eq. (48) since $\eta_{\mu}$ rapidly attenuates with time. This ensures that the typical magnitude of features in $\eta_{\mu}$ and $\mu_{\eta}$ are scaled, making it possible to control the spread of initial values for the real-time dynamics by reducing the variance of thermalization trajectories.

For example, for $r_{v \eta}=1$, the average magnitudes of $\eta_{v}^{\text {new }}$ and $v_{\eta}^{\text {new }}$ over a realization are approximately equal. Alternatively, $r_{\nu \eta}$ can be chosen to reduce the variance of $\operatorname{Tr}[\rho(t)]$ by reducing the magnitude of $v$ close to zero. However, in Sec. VI A we will show that taking this limit is not desirable as $\operatorname{Im}\left[\eta_{\nu}\right]$ grows with $r_{\nu \eta}$, resulting in numerical instability.

\section{DIFFERENT FORMS OF THE ESLN}

From Eq. (30), it is clear that the dynamics of each stochastic $\rho$ is not trace-preserving. This can lead to exponential blowup [42,43] of the trace and requires punitively large sample size for convergence. One way of enforcing trace preservation is to instead consider the trace-normalized density matrix, $\tilde{\rho}(t)=\rho(t) / \operatorname{Tr}[\rho(t)]$, satisfying [9]

$$
\begin{aligned}
i \hbar \frac{d \tilde{\rho}(t)}{d t}= & {[H(t), \tilde{\rho}(t)]-\eta(t)\left[\sigma_{z}, \tilde{\rho}(t)\right] } \\
& -\frac{\hbar}{2} v(t)\left\{\sigma_{z}-\sigma(t), \tilde{\rho}(t)\right\}
\end{aligned}
$$

where we have introduced the guide spin

$$
\sigma(t)=\frac{\operatorname{Tr}\left[\sigma_{z} \rho(t)\right]}{\operatorname{Tr}[\rho(t)]}=\operatorname{Tr}\left[\sigma_{z} \tilde{\boldsymbol{\rho}}(t)\right] .
$$

Simulating this normalized $\tilde{\rho}(t)$ still requires knowledge of the original $\operatorname{Tr}[\rho(t)]$ to perform the required statistical averaging since $\rho^{p h}(t)=\langle\rho(t)\rangle=\langle\tilde{\rho}(t) \operatorname{Tr}[\rho(t)]\rangle$. It is possible to overcome this problem via a transformation that enforces trace preservation for each realization while preserving the original ensemble mean $[6-8,44,45]$, i.e., $\rho^{p h}(t)=\langle\tilde{\rho}(t)\rangle$. Such a transformation of the probability measure, $\mathcal{W} \rightarrow \mathcal{W}^{\prime}$, is called a Girsanov transformation, where both the transformed and the original measures give rise to identical observables $[9,46-$ 48]. That is,

$$
\rho^{p h}(t)=\langle\rho(t)\rangle_{\mathcal{W}}=\langle\tilde{\rho}(t)\rangle_{\mathcal{W}^{\prime}},
$$

where $\langle\ldots\rangle_{\mathcal{W}}=\int d z_{1} d z_{2} \mathcal{W}\left[z_{1}, z_{2}\right] \ldots$ denotes the ensemble average over noises $z_{1}=\left(\eta \eta^{*} v v^{*}\right)^{T}$ and $z_{2}=\left(\mu \mu^{*}\right)^{T}$ drawn from the original Gaussian distribution $\mathcal{W}\left[z_{1}, z_{2}\right]$, and similarly $\langle\ldots\rangle_{\mathcal{W}^{\prime}}$ over noises $z_{1}^{\prime}, z_{2}^{\prime}$ drawn from the transformed distribution $\mathcal{W}^{\prime}\left[z_{1}^{\prime}, z_{2}^{\prime}\right]$, with $\tilde{\rho}(t)$ being evolved using the $z_{1}^{\prime}, z_{2}^{\prime}$ noises. This technique is well understood in the context of stochastic Schrödinger equations [6,49,50].

Performing a Girsanov transformation of the SLN Eq. (50), we arrive at an alternative equation of motion (see Appendix A for details) which we refer to as the guided SLN,

$$
\begin{aligned}
i \hbar \frac{d \rho(t)}{d t}= & {[H(t), \rho(t)]-\left[\eta(t)+\frac{i}{\hbar} \int_{0}^{t} d t^{\prime} K_{\eta v}\left(t-t^{\prime}\right) \sigma\left(t^{\prime}\right)\right] } \\
& \times\left[\sigma_{z}, \rho(t)\right]-\frac{\hbar}{2} v(t)\left\{\sigma_{z}-\sigma(t), \rho(t)\right\}
\end{aligned}
$$


noting that $\rho(t)$ is evolved rather than $\tilde{\rho}(t)$, with $\sigma(t)$ being the guide spin of Eq. (51). From Eq. (52), the physical density matrix is then obtained by averaging over realizations of this new guided dynamics.

Another equivalent strategy is to start from the traceviolating Eq. (24) and divide $\rho$ by its trace at each time step. When performing stochastic sampling, the trace still needs to be taken into account according to Eq. (A1). This can be avoided as shown above by shifting the mean of the $\eta$ noise which leads to the same Eq. (53) but without the guide term $\sigma(t)$ in the anticommutator,

$$
\begin{aligned}
i \hbar \frac{d \rho(t)}{d t}= & {[H(t), \rho(t)]-\left[\eta(t)+\frac{i}{\hbar} \int_{0}^{t} d t^{\prime} K_{\eta \nu}\left(t-t^{\prime}\right) \sigma\left(t^{\prime}\right)\right] } \\
& \times\left[\sigma_{z}, \rho(t)\right]-\frac{\hbar}{2} v(t)\left\{\sigma_{z}, \rho(t)\right\} .
\end{aligned}
$$

The physical density matrix is then obtained by the stochastic average $\rho^{p h}(t)=\langle\rho(t) / \operatorname{Tr}[\rho(t)]\rangle$, and we refer to this equation of motion as the normalized SLN.

To summarize, three forms of the SLN have been derived here:

(i) The original SLN, Eq. (24), which is not tracepreserving.

(ii) The guided SLN, Eq. (53), which preserves the trace via a Girsanov transformation.

(iii) The normalized SLN, Eq. (54), where the trace of the density matrix is explicitly normalized.

Alternatively, it is straightforward to derive all three (original, guided, and normalized) versions of the spin dynamics, Eqs. (27)-(30). For completeness, we give below their guided form, equivalent to Eq. (53):

$$
\begin{gathered}
\hbar \frac{d \sigma_{x}(t)}{d t}=-[\epsilon(t)-2 \hat{\eta}(t)] \sigma_{y}(t)-i \nu(t) \frac{\sigma_{x}(t) \sigma_{z}(t)}{\operatorname{Tr}[\rho(t)]}, \\
\hbar \frac{d \sigma_{y}(t)}{d t}=-\Delta \sigma_{z}(t)+[\epsilon(t)-2 \hat{\eta}(t)] \sigma_{x}(t)-i \nu(t) \frac{\sigma_{y}(t) \sigma_{z}(t)}{\operatorname{Tr}[\rho(t)]}
\end{gathered}
$$

$$
\hbar \frac{d \sigma_{z}(t)}{d t}=\Delta \sigma_{y}+i v(t) \operatorname{Tr}[\rho(t)]-i v(t) \frac{\sigma_{z}^{2}(t)}{\operatorname{Tr}[\rho(t)]},
$$

and $\operatorname{Tr}[\rho(t)]$ evolving according to Eq. (30), with $\hat{\eta}$ being simply the shifted $\eta$,

$$
\hat{\eta}(t)=\eta(t)+\frac{i}{\hbar} \int_{0}^{t} d t^{\prime} K_{\eta \nu}\left(t-t^{\prime}\right) \frac{\sigma_{z}\left(t^{\prime}\right)}{\operatorname{Tr}\left[\rho\left(t^{\prime}\right)\right]},
$$

having written the guide spin $\sigma(t)$ in the form given by Eq. (51). As before, the time-dependent spins here represent quantum averages over a single stochastic density matrix $\sigma_{i}(t)=\operatorname{Tr}\left[\sigma_{i} \rho(t)\right]$.

The same transformation has also recently been applied to density matrices starting in partitioned or pure states and evolved via the SLN [7-9], though the reasoning was slightly different, thermalization was not included, and no numerical results were shown. The authors started from the original SLN and applied the transformation

$$
\tilde{\rho}(t)=\rho(t) \exp \left\{\frac{i}{\hbar} \int_{0}^{t} d t^{\prime} v\left(t^{\prime}\right) \gamma\left(t^{\prime}\right)\right\}
$$

with $\gamma(t)$ being an unknown function. $\gamma(t)$ was later chosen to enforce trace-preserving dynamics, leading to the obvious choice $\gamma(t)=\sigma(t)$ and an equation identical to Eq. (53). Following the same steps as above, the exponential factor in the sampling procedure is removed to arrive at the simple averaging of the trajectories.

\section{STOCHASTIC DIFFERENTIAL EQUATIONS}

It is well known that Langevin equations are ill defined when expressed as differential equations due to the white noise being everywhere discontinuous [46,51-55]. Instead, discretized integral equations involving the Wiener process increment are used to bring them into a well-defined form. The standard result for a set of coupled SDEs of a vector of functions $\boldsymbol{\rho}_{h}=\left\{\rho_{h}^{k}\right\}$ is

$$
d \rho_{h}^{k}=a^{k}\left(t_{h}, \boldsymbol{\rho}_{h}\right) d t+\sum_{j} B^{k j}\left(t_{h}, \boldsymbol{\rho}_{h}\right) d W_{h}^{j},
$$

where $\boldsymbol{a}\left(t_{h}, \rho_{h}\right)$ is the deterministic (so-called drift) component of the dynamics and the index $h$ is associated with the discrete proper time $t_{h}=h d t . \boldsymbol{B}\left(t_{h}, \boldsymbol{\rho}_{h}\right)=\left\{B^{k j}\left(t_{h}, \boldsymbol{\rho}_{h}\right)\right\}$ is a matrix whose rows $\boldsymbol{b}^{k}\left(t_{h}, \boldsymbol{\rho}_{h}\right)$ are vectors associated with each $\rho_{h}$, and $d W_{h}^{j}=W_{h+1}^{j}-W_{h}^{j}$ is the Wiener increment, where $W_{h}^{j}=\int_{0}^{t_{h}} d t^{\prime} x_{j}\left(t^{\prime}\right)$, with $x$ being a white noise. This is just a first-order Taylor expansion known as the Euler-Maruyama approximation or the Cauchy-Euler method [54], for which the deterministic and stochastic Taylor expansions are the same.

For a higher-order scheme, additional terms that do not appear in the deterministic Taylor expansion arise from the application of stochastic calculus in either Stratonovich or Itô form [53]. For example, the second-order Itô scheme, known as the Milstein scheme, reads

$$
\begin{aligned}
\rho_{h+1}^{k}= & \rho_{h}^{k}+a^{k}\left(t_{h}, \boldsymbol{\rho}_{h}\right) d t+\sum_{j} B^{k j}\left(t_{h}, \boldsymbol{\rho}_{h}\right) d W_{h}^{j} \\
& +\sum_{l} \sum_{j_{1}, j_{2}} B^{l j_{1}}\left(t_{h}, \boldsymbol{\rho}_{h}\right) \frac{B^{k j_{2}}\left(t_{h}, \boldsymbol{\rho}_{h}\right)}{\partial \rho_{h}^{l}} I_{j_{1}, j_{2}},
\end{aligned}
$$

where

$$
I_{j_{1}, j_{2}}=\int_{t_{h}}^{t_{h+1}} \int_{t_{h}}^{t_{h+1}} d W_{h}^{j_{1}} d W_{h}^{j_{2}}
$$

is the Wiener integral. The solutions to these integrals grow in complexity as the number of noises and/or the system size increases, though general solutions are known [52]. In addition, the normal rules of calculus do not apply in Itô calculus but do for Stratonovich, at the cost of introducing a correction which modifies the deterministic drift [54]. For the purposes of this work, where many noises are necessary, Stratonovich calculus is more computationally efficient with easier implementation and hence this interpretation will be used. The dynamics still has the same form as Eq. (59), but the drift $a^{k}\left(t_{h}, \rho_{h}\right)$ is replaced by the modified drift

$$
\tilde{a}^{k}\left(t_{h}, \boldsymbol{\rho}_{h}\right)=a^{k}\left(t_{h}, \boldsymbol{\rho}_{h}\right)-\frac{1}{2} \sum_{l j} B^{l j}\left(t_{h}, \boldsymbol{\rho}_{h}\right) \frac{\partial B^{k j}\left(t_{h}, \boldsymbol{\rho}_{h}\right)}{\partial \rho_{h}^{l}} .
$$


Since Stratonovich SDEs obey the rules of ordinary calculus, a family of Runge-Kutta numerical methods can be developed. We shall use a Heun scheme [56] with strong order convergence of 1.0 compared to only 0.5 for the naive Euler-Maruyama approximation [53], making it the same as the second-order Itô-Milstein scheme [57]. The Heun scheme uses an intermediary prediction step to calculate a supporting value $\hat{\boldsymbol{\rho}}_{h+1}$ which improves on an initial guess, so that the next time step prediction becomes

$$
\begin{aligned}
\rho_{h+1}^{k}= & \rho_{h}^{k}+\frac{1}{2}\left[\tilde{a}^{k}\left(t_{h}, \boldsymbol{\rho}_{h}\right)+\tilde{a}^{k}\left(t_{h}, \hat{\boldsymbol{\rho}}_{h+1}\right)\right] d t \\
& +\frac{1}{2} \sum_{j}\left[B^{k j}\left(t_{h}, \boldsymbol{\rho}_{h}\right)+B^{k j}\left(t_{h}, \hat{\boldsymbol{\rho}}_{h+1}\right)\right] d W_{h}^{j},
\end{aligned}
$$

where the supporting value $\hat{\boldsymbol{\rho}}_{h+1}$ is obtained via an EulerMaruyama integrator with the Stratonovich correction,

$$
\hat{\rho}_{h+1}^{k}=\rho_{h}^{k}+\tilde{a}^{k}\left(t_{h}, \boldsymbol{\rho}_{h}\right) d t+\sum_{j} B^{k j}\left(t_{h}, \boldsymbol{\rho}_{h}\right) d W_{h}^{j} .
$$

The derivation of the final Stratonovich corrections in imaginary and real time are provided in Appendix B. We give there the explicit form of the Heun scheme of Eq. (63) for the spin-boson model in terms of the components of the density matrix, as well as for mean $x, y$, and $z$ spins. Note that there is no correction for the trace.

The final procedure for the numerical solution of the ESLN is as follows:

(1) Generate the appropriate filtering kernels $G(t)$, Eqs. (44)-(47), from the model-specific physical kernels $K(t)$, Eqs. (15)-(18), via application of the discrete Fourier transform and its inverse.

(2) For each new realization of the stochastic density matrix, generate a set of orthogonal noise components $\eta_{\eta}, \eta_{\nu}, \eta_{\mu}, v_{\eta}, \mu_{\eta}$, and $\mu_{\mu}$.

(3) Rescale the $\eta_{\nu}, v_{\eta}$ and $\eta_{\mu}, \mu_{\eta}$ noises as required, as detailed in Sec. III B.

(4) Initialize the prethermalized density matrix in the state $\bar{\rho}(\tau=0)=\mathbb{I}$ before evolving in imaginary time for $\tau \in$ $[0, \beta \hbar]$, using the Stratonovich modified drift as detailed in Appendix B, Eq. (B19).

(5) Initialize the real-time stochastic density matrix using the final value from the imaginary-time evolution, $\rho\left(t_{0}\right)=$ $\bar{\rho}(\beta \hbar)$. Evolve it in real time with the Stratonovich modified drift, Eq. (B14) in Appendix B. If desired, one of the trace-preserving variants of Eqs. (53) and (54) may be used. Alternatively, spin dynamics given by Eqs. (27)-(30) can be used instead, with the corresponding Stratonovich corrections, Eqs. (B15)-(B17).

(6) Repeat the simulation (points 4 and 5) as many times as required, before taking the ensemble average over the realizations of the density matrix, then divide by the value of the trace of the ensemble average after thermalization $\operatorname{Tr}\left[\left\langle\rho\left(t_{0}\right)\right\rangle\right]$ to obtain the physical density matrix.

\section{RESULTS}

\section{A. Noise and convergence}

Using the noise generation procedure detailed in Sec. III where the noise components are generated in Fourier space before taking the inverse Fourier transform, it is found that the required correlation functions (Fig. 1) are satisfied and converge well. The cross-correlated noise $\eta_{\mu}$ presents a computational bottleneck in terms of simulation time, since Fourier methods cannot be employed and weighted sums of whitenoise random numbers must be computed directly. Choosing $\eta_{\mu}$ as a colored noise and $\mu_{\eta}$ as a white noise also reduces the rate at which the cross-time correlation matrix converges with sample size, making the cross-correlated noise generation doubly expensive [14]. No alternative choice is known to us at the time of writing.

Next we discuss the importance of the higher-order numerical scheme (Heun) considered in Sec. V (and derived in Appendix B) in solving the SDEs. To this end, we shall consider the real-time dynamics of $\left\langle\sigma_{z}(t)\right\rangle$ for a constant spinboson Hamiltonian, initialized in the proper thermal state. In Fig. 2, we compare the convergence properties of $\left\langle\sigma_{z}(t)\right\rangle$ for increasing sample size using both the Euler-Maruyama and Heun discretization schemes.

The expected result is for the spin to remain constant and equal to the value obtained during thermalization $(t=$ 0 ) during all real times $t \geqslant 0$; this behavior is only evident for sufficiently large sample size. It is clear that the error depends almost entirely on the properties of the noises rather than inclusion of higher-order dynamical terms coming from stochastic calculus, since the results obtained using the Heun scheme are indistinguishable from the Euler-Maruyama scheme. This indicates that the convergence is solely statistical, depending almost entirely on the sample size. However, in the special case of weak coupling being simulated out to late times when the statistical convergence is well controlled, the Heun scheme is necessary. If the Euler-Maruyama scheme is used, the coherences ( $x$ and $y$ spins) oscillate within an exponentially growing envelope at late times, whereas the Heun scheme reduces the time-stepping error sufficiently to recover decoherent dynamics. For this reason we use the Heun scheme for all subsequent results, but note that future work should focus on optimizing the noise generation method for better convergence instead of improving the discretization scheme. The Stratonovich corrections of Eqs. (B15) and (B16) are also used in all subsequent results for completeness, though their effect is negligible. This is unsurprising since they are of order $O(d t)$. Finally, the $\eta-\eta$ correlation in the inset in Fig. 2 uses the same noises as the dynamics, averaged over the same number of runs, emphasizing the equivalence between the convergence of the noise correlations with the convergence of the sample dynamics.

Though the correlation functions can be obtained for any choice of scaling $r_{\mu \eta}$ and $r_{\nu \eta}$ introduced in Sec. III B, choosing $r_{\nu \eta}$ to minimize the growth of the trace should extend the time accessible by simulation. The effect of increasing $r_{\nu \eta}$ from 0.1 to 5 on the standard error of the mean $\operatorname{trace} \operatorname{Tr}\left[\left\langle\rho\left(t_{\max }\right)\right\rangle\right]$ for a sample of 10000 realizations is shown in Fig. 3 for two different environment coupling strengths $\alpha$. It is tempting to take the limit where $r_{\nu \eta}$ becomes large and $v \rightarrow 0$ so that the dynamics becomes exactly trace preserving, see Eq. (30), but such a choice would cause $\eta_{v}$ to be very large, leading to poor convergence or instability. We see that the order of magnitude of the error increases rapidly beyond a narrow band of ratios for which it is at a minimum around $r_{\nu \eta} \approx 0.5$, evidence that 

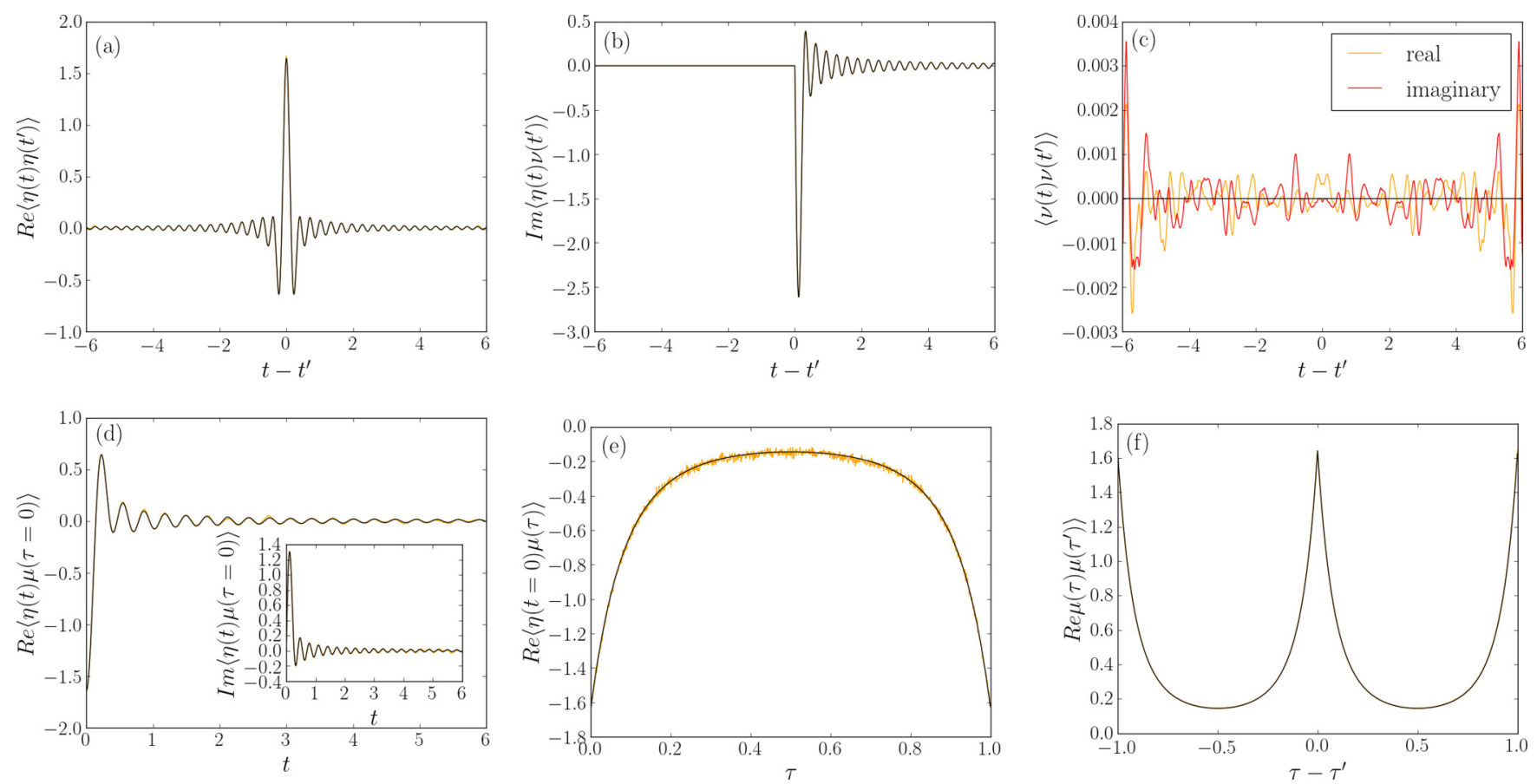

FIG. 1. Correlation functions of the noises, where the black line is the appropriate kernel given in Eqs. (15)-(19) and the orange line is the numerical correlation computed for 1 million realizations with $\beta=1, t_{\max }=6, d t=d \tau=10^{-3}$, and $\omega_{c}=20$. If only the black curve is visible, the orange curve lies exactly underneath. All other correlations (not shown) are zero to within 0.001 . (a) The $\eta-\eta$ autocorrelation. (b) The $\eta-v$ correlation. (c) The $\nu-\nu$ autocorrelation, which is zero as required (within the adopted precision). (d) The real part of the $\eta-\mu$ correlation when $\tau=0$, with the imaginary part given in the inset. (e) The $\eta-\mu$ correlation when $t=0$. (f) The $\mu-\mu$ autocorrelation. Optimal scaling of $r_{v \eta}=0.5$ with $r_{\mu \eta}=1$ has been used in all cases.

the convergence of the system dynamics is very sensitive to the properties of the noises even when they satisfy the necessary correlation functions. In all subsequent results, scalings

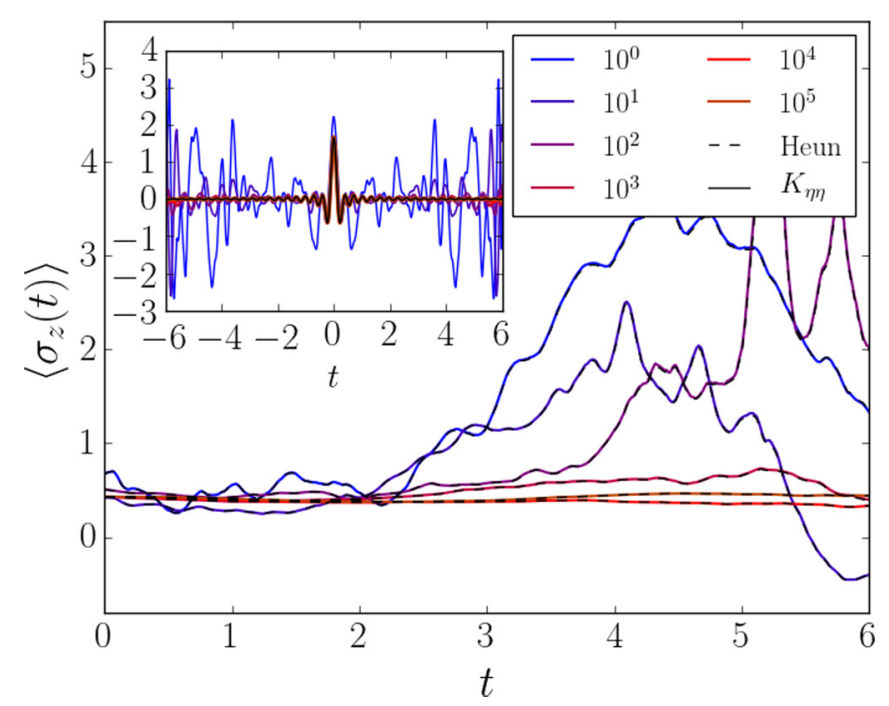

FIG. 2. Convergence of $\left\langle\sigma_{z}(t)\right\rangle$ for a constant Hamiltonian with $\Delta=1$ and $\epsilon=-1$ for the Euler-Maruyama (solid colored lines) and Heun (dashed black lines) schemes for the same sets of parameters, both performed for a range of sample sizes and using the original SLN of Eq. (24). The inset shows the $\eta-\eta$ correlation for the same range of sample sizes, as well as the corresponding physical kernel $K_{\eta \eta}\left(t-t^{\prime}\right)$ (black line). $\beta=1, t_{\max }=6, d t=d \tau=10^{-3}, \alpha=$ $0.05, \omega_{c}=20, r_{v \eta}=0.5$, and $r_{\mu \eta}=1$. of $r_{\nu \eta}=0.5$ and $r_{\mu \eta}=1.0$ are used. While similar optimal scaling $r_{\mu \eta}$ could be chosen to minimize the spread of initial values from thermalization, the variance is not significant and the simpler choice of $r_{\mu \eta}=1.0$ is sufficient. It is also clear that increasing the coupling strength $\alpha$ makes the convergence worse as expected, since the noise amplitudes scale like $\sqrt{\alpha}$.

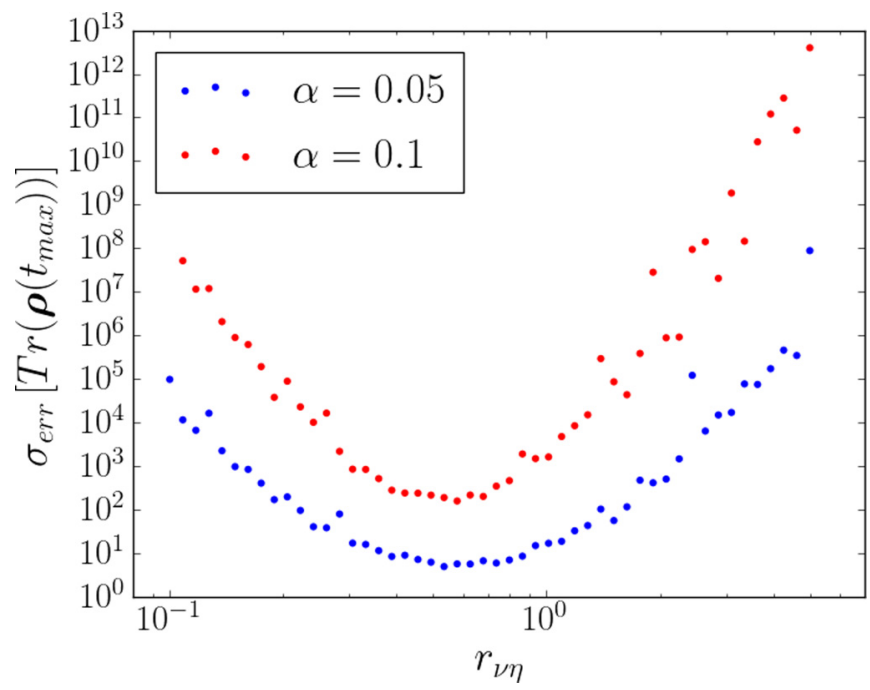

FIG. 3. The standard error of the mean $\operatorname{Tr}\left[\rho\left(t_{\max }\right)\right]$ at its final time step for several values of the scaling factor $r_{v \eta}$. For each scaling factor, 10000 runs for real-time dynamics were performed. $\beta=$ $1, t_{\max }=10, d t=d \tau=10^{-3}, \omega_{c}=20$, and $\epsilon=\Delta=0$. In this case, the minimizing value of $r_{v \eta}$ is $\approx \frac{1}{2}$. 

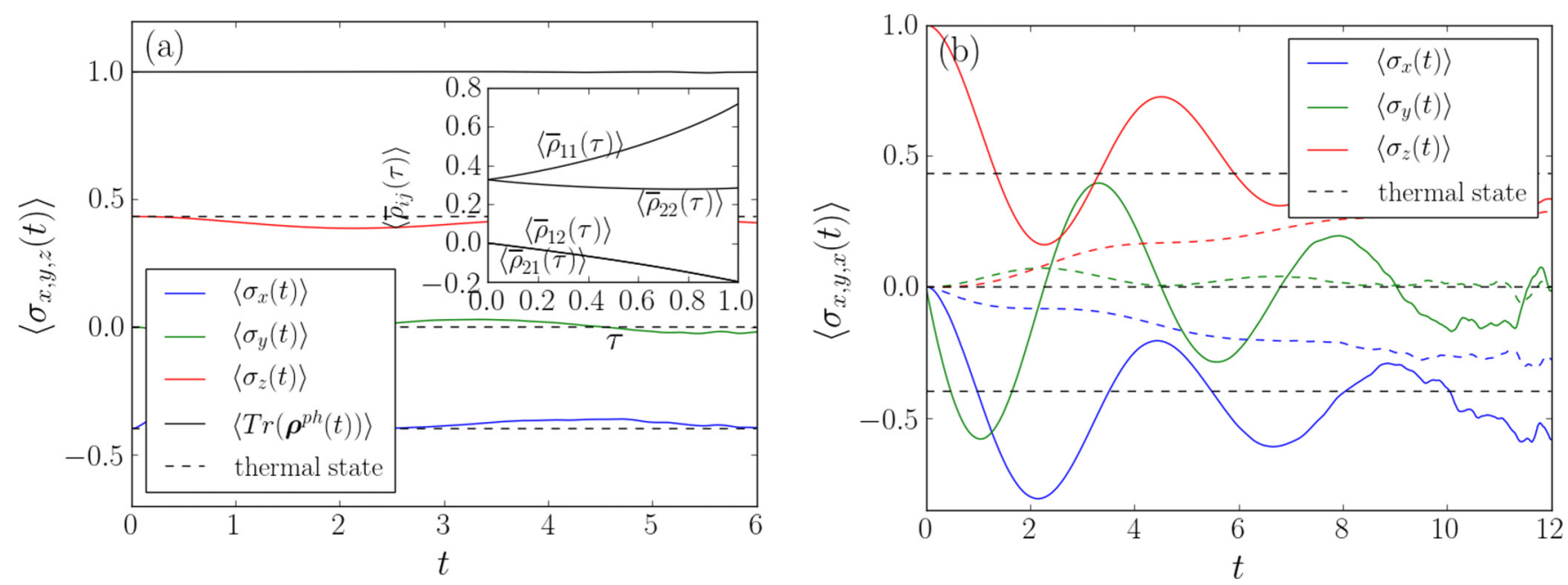

FIG. 4. Physical spins evolved by means of the original SLN, Eq. (24), for different initial conditions using a constant Hamiltonian with $\Delta=1$ and $\epsilon=-1$. Other simulation parameters are $\beta=1, t_{\max }$ as shown, $d \tau=d t=10^{-3}, \alpha=0.05$, and $\omega_{c}=20$. Dashed black lines are the values of the thermalized spins obtained from the end of imaginary-time evolution. (a) Each realization was initialized in the canonical equilibrium state obtained from thermalization in imaginary time and averaged over 10 million runs. Inset: The elements of the density matrix during imaginary-time evolution. (b) Initially decoupled from the environment and initialized out of equilibrium, the spin components all decay toward the correct canonical equilibrium state (black dashed lines) as obtained separately from thermalization. 100 million realizations were used. Since there was no thermal preparation, the $\eta$ noise has no $\eta_{\mu}$ component. Spins initialized in the pure initial state $\sigma_{z}(0)=1, \sigma_{x}(0)=$ $\sigma_{y}(0)=0$ are given by the solid colored lines, while the zero initial state $\sigma_{x}(0)=\sigma_{y}(0)=\sigma_{z}(0)=0$ spins are given by dashed colored lines.

Unlike the noise amplitudes, the variance within a sample grows nonlinearly with $\alpha$ rather than $\sim \sqrt{\alpha}$.

\section{B. Thermalization}

The ESLN is unique in its ability to simulate quantum dynamics exactly, starting in the canonical equilibrium state with system-environment entanglement arising from joint preparation. In Fig. 4(a), stationary-state dynamics for the spin-boson system is shown using the original SLN of Eq. (24), with the open system having been initialized in the thermal state via evolution in imaginary time [Eq. (4)]. Small-amplitude oscillations around the equilibrium state are observed, most likely caused by variation in the initial condition arising from the stochastic nature of thermalization, and vanishing as the sample size increases. For completeness, the elements of the prethermalized density matrix $\langle\overline{\boldsymbol{\rho}}(\tau)\rangle$ are included in the inset, being evolved in $\tau$ from the initial unitary state at $\tau=0$ to the thermal state at $\tau=\beta \hbar$. This is the physical expectation obtained by the ensemble average over many realizations of the environment noises, divided by the final trace after averaging. The physical trace is divided by $\operatorname{Tr}\langle\overline{\boldsymbol{\rho}}(\beta \hbar)\rangle$ to ensure that $\operatorname{Tr}\left[\rho^{p h}\left(t_{0}\right)\right]=1$.

It is also necessary to check that the system decays to the correct thermal state after being initially partitioned from the environment. In Fig. 4(b), the open system was initialized in the pure state $\rho_{i j}(0)=\delta_{i 1} \delta_{j 1}$ (solid lines), corresponding to $\sigma_{z}(0)=1$ and $\sigma_{x}(0)=\sigma_{y}(0)=0$. In another simulation (dashed lines) the density matrix was initialized in the halfhalf state $\rho_{11}=\rho_{22}=\frac{1}{2}$ and $\rho_{12}=\rho_{21}=0$, which corresponds to the spin-zero state $\sigma_{x}(0)=\sigma_{y}(0)=\sigma_{z}(0)=0$. In both cases, the coupling to the environment was switched on at $t=0$ so that the system then begins to thermalize. Clearly, when initialized in both the pure state $\sigma_{z}(0)=1$ (colored solid lines) and the $\sigma_{z}(0)=0$ state (colored dashed lines), the spins decay toward the thermal state as obtained from imaginarytime evolution (black lines) in the manner expected.

\section{Forms of the ESLN}

Since each realization of the trace undergoes noisy growth within an exponential envelope, Eq. (30), such that the average trace converges poorly (see Fig. 3), it may be desirable to use one of the trace-preserving variants of the ESLN: the guided ESLN of Eq. (53) or the normalized ESLN of Eq. (54). In both cases, the physical trace after the ensemble average should be constant. As for individual realizations, in the case of guided dynamics, the trace is preserved exactly since the guide spin forces the derivative of the trace to be zero. For individual realizations of the normalized dynamics, however, the trace is not required to be constant and the ensemble average is taken over $\rho(t) / \operatorname{Tr}[\rho(t)]$ rather than over $\rho(t)$, forcing the physical trace to be 1 .

Figure 5(a) shows example dynamics for a single realization of the $z$ spin evolved using the guided ESLN of Eq. (53). In Fig. 5(b), the spins are evolved using the normalized ESLN of Eq. (54), and the guided ESLN for comparison and averaged over an ensemble of 1000 realizations. A single realization of the $z$ spin for the original ESLN of Eq. (24) can be found in Fig. 2, and an ensemble average in Fig. 4(a); the size of the ensemble average is not the same as in Fig. 5(b), but this does not affect the point being made here. In the case of the normalized ESLN, the trace of a single spin trajectory is not required to be constant or even positive at all times. Since the trace is always initially positive, there are individual realizations where the trace crosses zero and becomes negative. Since the ensemble average is taken over $\rho(t) / \operatorname{Tr}[\rho(t)]$, the physical density matrix and its observables will exhibit large 

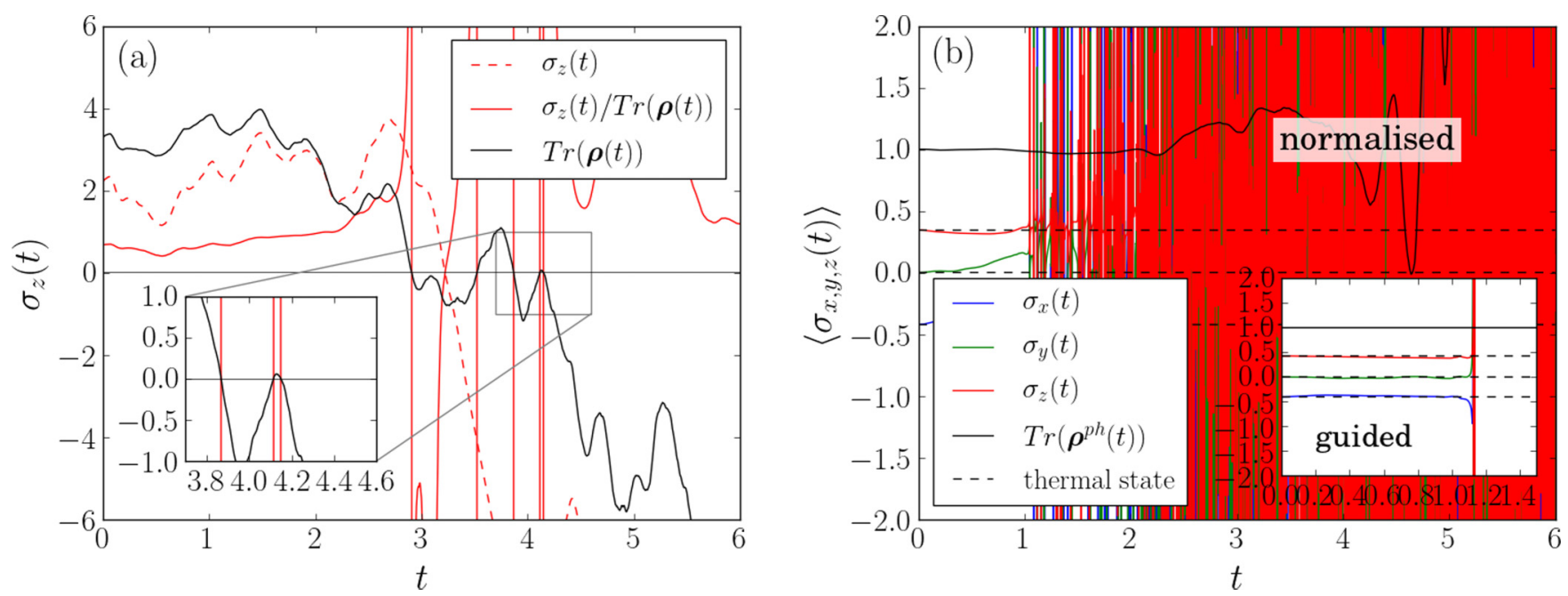

FIG. 5. (a) A single realization of the normalized ESLN spin dynamics, where $\sigma_{z}(t)$ is the $z$ spin, evolved by Eq. (54). The inset highlights the behavior when $\operatorname{Tr}[\rho(t)]$ crosses zero. The magnitude of the spikes in $\sigma_{z}(t) / \operatorname{Tr}[\rho(t)]$ at these points reaches $\approx 1300$. (b) 1000 realizations of the normalized (main figure) and guided (inset) ESLN spin dynamics. In all cases $\beta=1, t_{\max }$ as shown, $d t=d \tau=10^{-3}, \Delta=1, \epsilon=$ $-1, \alpha=0.05$, and $\omega_{c}=20$ were used.

(infinite) spikes whenever $\operatorname{Tr}[\rho(t)]=0$; however, in practice it is unlikely that the trace would ever be exactly zero so the spikes remain finite. Figure 5(a) is an example of such a pathological trajectory. As a result, even a small sample of 1000 realizations as in Fig. 5(b) accumulates many spikes, completely destroying the physical dynamics. The averaged trace in Fig. 5(b) also fails to be constant, since individual realizations of the trace are computed directly [see black line in Fig. 5(a)] and their averages are obtained in the normal way. The variation in the average trace is thus an indication of undersampling only, whereas the rapid fluctuation of the spins is largely independent of the sampling, arising only from this division by (nearly) zero.

Individual realizations of the spins and trace evolved via the guided ESLN are qualitatively similar to those evolved by the normalized ESLN, with the exception that the guided trace is constant by definition; it is not simulated directly but remains at its initial value $\operatorname{Tr}[\bar{\rho}(\beta \hbar)]$. This is true even when $\rho(t)$ is simulated rather than the spins and trace, in which case $\rho_{11}+\rho_{22}$ stays constant to within $\pm 10^{-13}$ of its initial value. However, the guided ESLN includes a term containing the guide spin of Eq. $(51), \sigma(t)=\operatorname{Tr}\left[\sigma_{z} \rho(t)\right] / \operatorname{Tr}[\rho(t)]=$ $\sigma_{z}(t) / \operatorname{Tr}[\rho(t)]$, in which the $z$ spin is divided by the trace. This is just as pathological as taking the ensemble average of $\rho(t) / \operatorname{Tr}[\rho(t)]$ rather than $\rho(t)$ in the normalized ESLN, since the guide introduces the (possibly infinite) spikes directly into the dynamics of individual trajectories. The system is usually unable to recover, with individual realizations of the spins exceeding the maximum allowed integer size of $2^{63}-1$. The ensemble average similarly diverges, after which time the expectation values cease to be physically meaningful. An example for a sample of 1000 realizations is shown in the inset in Fig. 5(b). For both the guided ESLN and the normalized ESLN in Fig. 5, the breakdown occurs at $t \sim 1$.1. This feature is intrinsic to the equations of motion themselves, and cannot be removed using a larger sample since the probability of including a trajectory where a spike occurs at $t \leqslant 1.1$ increases with sample size.
Such behavior occurs regardless of whether the equations of motion for the density matrix or the spins are used, and does not appear to depend on the parameters chosen in any meaningful way. While Eqs. (53) and (54) with their corresponding ensemble averages analytically describe the correct physical dynamics, the averages appear to be valid only in the limit that the sample size is infinite-that is, for the analytic path integral of the distribution $\mathcal{W}$ over the noise variables $z_{1}, z_{2}$, rather than a statistical average as is practically obtained, for which the results are pathological. Thus improvements in convergence to address the growth of the trace must be obtained via other methods, such as exploiting or even optimizing the generation of the driving noises [14].

Concluding, both trace-conserving ESLN variants result in a pathological behavior in the dynamics that in practice cannot be cured by increasing the sample size. Hence, in the following, only the original SLN, Eq. (24), is used.

\section{Landau-Zener sweep}

\section{Modified limit for finite-temperature coupling}

In Fig. 6, the spin-boson system is linearly driven from negative to positive $\epsilon$ by a Landau-Zener (LZ) sweep for a range of inverse temperatures $\beta \in[0.1,5.0]$ [panel (a)] and environment coupling strengths $\alpha \in[0.01,0.05]$ [panel (b)]. The analytic LZ limit of Eq. (26) is valid for a spin which was initialized at zero temperature in its ground state in the infinite past, $\sigma_{z}(-\infty)=1$, with all other spins being zero. This limit describes the asymptotic state as $t \rightarrow \infty$ and while it was originally derived for an isolated spin [36], the result is valid for a zero-temperature dissipative spin as well $[11,38,39,41]$ so is often used as a numerical test for approximate methods $[8,9,11,27,39,40]$.

In practice, the spin is initialized with $\sigma_{z}\left(t_{0}\right)=1$ at some finite time in the past $t_{0}<0$ instead, rather than when $t_{0} \rightarrow$ $-\infty$. This causes the late-time dynamics to approach a slightly different limit $\left\langle\sigma_{z}\right\rangle_{L Z}^{t_{0}}$ that deviates from the asymptotic limit, approaching $\left\langle\sigma_{z}\right\rangle_{L Z}$ only as $t_{0} \rightarrow-\infty$. This can 

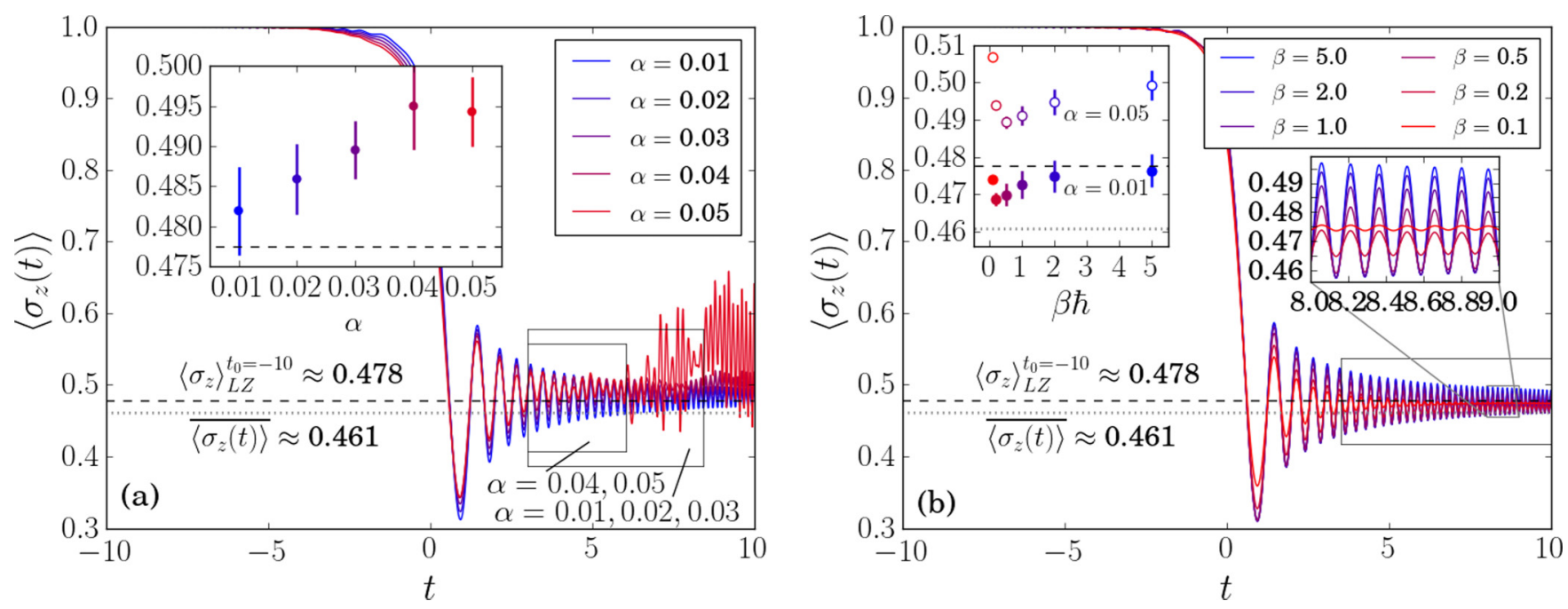

FIG. 6. Evolution of the physical $z$ spin $\left\langle\sigma_{z}(t)\right\rangle$ under LZ driving $\epsilon(t)=\kappa t$ with $\kappa=5$. The black dotted and dashed lines are the original and modified (for $t_{0}=-10$ ) LZ limits, respectively. (a) Dynamics for a range of coupling strengths $\alpha \in$ [0.01, 0.05] color coded from blue to red with increasing $\alpha$, all with the same temperature $\beta=1$, are shown. The inset shows the observed asymptotic value for each coupling using the same colors, obtained using 12 equally temporally spaced independent estimates of the mean for $\alpha=0.01,0.02,0.03$ over 1 million realizations and 6 equally spaced independent estimates of the mean for $\alpha=0.04,0.05$ over 10 million realizations, taken over the regions indicated by the labeled boxes. (b) Dynamics for a range of inverse temperatures $\beta \in[0.1,5.0]$, color coded from blue to red with increasing temperature. As before, the inset on the left shows the observed asymptotic value of the results using the same colors, obtained using 20 independent estimates of the mean over the boxed region. The solid circles are for the data shown in the main figure with $\alpha=0.01$, while the empty circles are for stronger coupling of $\alpha=0.03$ that remained well converged throughout the simulation. The inset on the right shows the detailed dynamics for times $8 \leqslant t \leqslant 9$ (see text). All other simulation parameters are $d t=d \tau=10^{-3}, \Delta=1$, and $\omega_{c}=20$.

clearly be seen in Fig. 7, where the deviation from the analytical limit is calculated for the isolated system (no coupling to the bath, $\alpha=0$ ) for many values of $t_{0}$. Thus Fig. 7 acts as a form of approximate calibration of the simulations with the bath coupling turned on, allowing us to modify the LZ limit

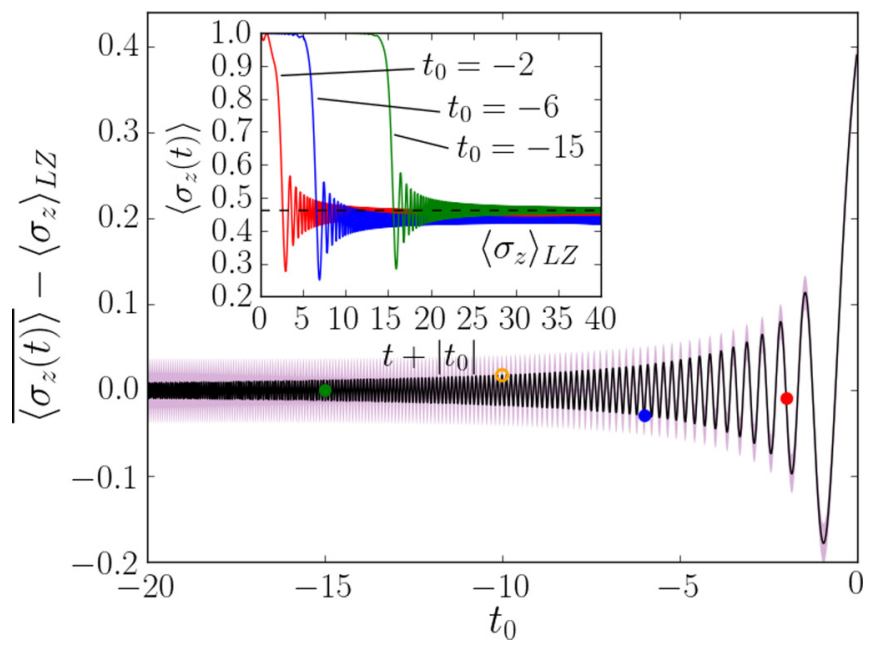

FIG. 7. The deviation of $\sigma_{z}(t)$ from the exact LZ limit for an isolated spin (no bath, $\alpha=0$ ) with initial preparation $\sigma_{z}\left(t_{0}\right)=1$. The error on the average is the filled pink area, with the average taken over the time period from the first maximum after the minimum in the inset to the end of the simulation. The solid points are colored to correspond to the used $t_{0}$ values for the evolution examples given in the inset. $t_{0}=-10$ is also highlighted (orange circle) since this is the $t_{0}$ used in subsequent results. $d t=d \tau=10^{-3}, \Delta=1, \epsilon(t)=\kappa t$, with $\kappa=5$. using the value obtained for the isolated system to account for the finiteness of $t_{0}$. Note however that this calibration alone is not sufficient to fully correct the limit for finite $\beta$ and nonzero $\alpha$, as Fig. 7 was obtained for the closed system with no bath rather than for an open system at zero temperature.

Using Fig. 7, we find that the deviation from the modified limit $\left\langle\sigma_{z}\right\rangle_{L Z}^{t_{0}}$ (shown by the dashed line) for a system coupled to a finite-temperature bath is larger for stronger coupling. This can been seen in Fig. 6(a). It is apparent that for the largest coupling $\alpha=0.05$, the required ensemble size becomes larger than the 10 million realizations used here, which for the reasonably long simulation time $-10 \leqslant t \leqslant 10$ takes $\sim 15$ hours on 360 CPUs, compared to only $\sim 1$ hour for 1 million realizations. As such, a smaller box has to be taken for higher coupling when calculating the mean $z$ spin as an estimate of the observed asymptote; see Fig. 7(a). This poor convergence may explain the otherwise anomalous mean value for $\alpha=$ 0.05 in the inset, which moves toward the shifted limit rather than away from it.

If the modified LZ limit $\left\langle\sigma_{z}\right\rangle_{L Z}^{t_{0}}$ for $t_{0}=-10$ had not been used, the observed asymptotes would never approach the original LZ limit, not even in the $\alpha \rightarrow 0$ limit. However, by using the modified limit we recover the expected asymptotic dynamics for small $\alpha$ while stronger coupling forces the $z$ spin away from the limit. This can be understood in terms of the renormalized tunneling matrix element [58],

$$
\Delta_{r}=\Delta\left(\frac{\Delta}{\omega_{c}}\right)^{\frac{\alpha}{1-\alpha}},
$$

which depends on the ratio $\Delta / \omega_{c}$. In this work where $\Delta / \omega_{c}<$ $1, \Delta_{r}$ decreases with the coupling strength. After $t=0$, the 
$\sigma_{z}=-1$ state becomes the lower energy state with both thermal fluctuations and tunneling contributing to transitions out of the initial $\sigma_{z}=+1$ state. Since the renormalized tunneling element $\Delta_{r}$ decreases with $\alpha$, the system is less likely to tunnel from $\sigma_{z}=+1$ to $\sigma_{z}=-1$, resulting in the observed increase in $\left\langle\sigma_{z}\left(t \lesssim t_{\max }\right)\right\rangle$.

In Fig. 6(b) we examine the behavior of the limit for a range of inverse temperatures $\beta \in[0.1,5.0]$, and again find that the modified limit is required to observe the expected asymptotic results; the original limit is missed altogether. As the temperature is decreased, the observed asymptote tends toward the modified limit as expected, with strange behavior for high temperatures (see inset, discussed below). Consistent with Fig. 6(a), increasing the coupling to $\alpha=0.03$ (empty circles) from $\alpha=0.01$ (solid circles) in the inset has the effect of lifting the observed asymptote, though the exact scaling of this shift for different $(\alpha, \beta)$ pairs has not been investigated as it is not of interest to us here.

For medium to high temperatures $0.5 \leqslant \beta<2$, the asymptotic $z$ spin decreases. This is as expected, since thermal fluctuations in the bath serve to destroy coherence, with the mean of all the spin components being zero in the hightemperature limit. Strangely, for very high temperatures $\beta<$ 0.5 , the $z$ spin increases toward the modified limit before surpassing it altogether. This is not caused by poor statistical convergence, as is shown in the magnified inset between $t=8$ and 9 where the position of the curves clearly increases for the two hottest temperatures. We suggest that this rapid increase in the observed asymptote for higher temperatures occurs as the energy scale of thermal fluctuations in the bath approaches the typical energy separation between the two states at the end of the simulation, $\epsilon\left(t_{\max }\right)$, providing enough energy for the system to jump into the higher energy state. This is not a true asymptotic effect, but a transient effect in the window $0<t \lesssim t_{\max }$ that should vanish as $t \rightarrow \infty$. The dimensionless energy ratio $q$ between the thermal energy scale of the bath, $k_{B} T$, and the energy separation between the states, $\hbar \epsilon\left(t_{\max }\right)$ (setting $\hbar=k_{B}=1$ ),

$$
q=\frac{k_{B} T}{\hbar \epsilon\left(t_{\max }\right)}=\frac{1 / \beta}{\epsilon\left(t_{\max }\right)},
$$

will be of order 1 when thermal fluctuations are large enough to overcome the finite bias within the simulation window. For the hottest temperature in Fig. 6(b) $(\beta=0.1)$ the thermal energy scale is $\sim 10$ and the energy separation is $\sim 50$ so that $q=0.2$. While not of order 1 , an observable increase in the mean spin would be expected, though the observed prominence of the high-temperature increase in the spin remains surprising.

\section{Thermalization to recover the original limit}

It is possible to circumvent the need for a modified LZ limit altogether by initializing the $z$ spin to be closer to the true LZ spin at the actual finite (negative) $t_{0}$, rather than being equal to one at $t_{0}$. The true LZ spin is initialized with $\sigma_{z}(-\infty)=1$ in the infinite past when the bias was infinitely large. It is obvious that the change in spin acquired during its evolution from $-\infty$ up to the finite time $t_{0}$ would be different from $\sigma_{z}\left(t_{0}\right)=1$, which is commonly taken as the initial condition at

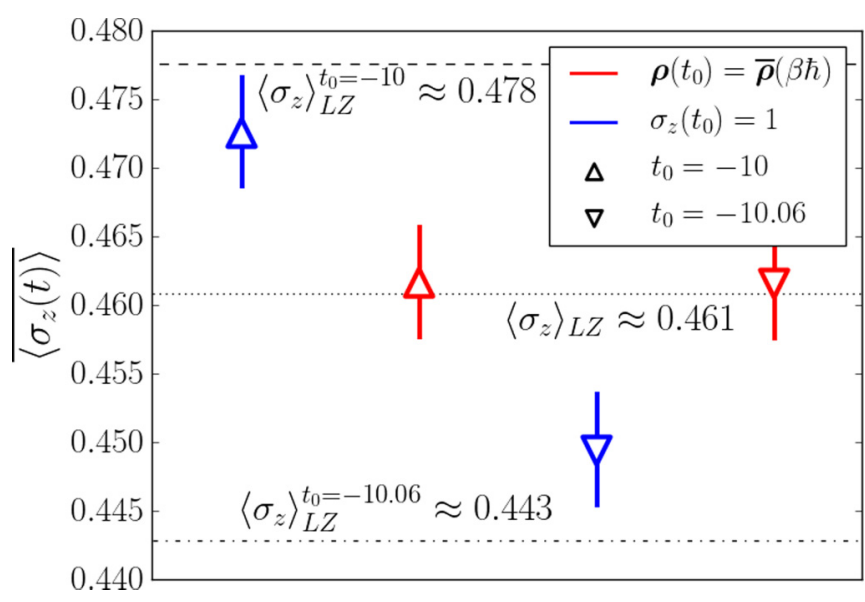

FIG. 8. Observed mean asymptotic $z$ spin when initialized with $\sigma_{z}\left(t_{0}\right)=1$ and all other spin components zero (blue) and when the system is initially thermalized in accordance with the initial value of the bias $\epsilon\left(t_{0}\right)$ (red). Two different values of $t_{0}$ are shown, specifically chosen so that one shifts the LZ limit upward $\left(t_{0}=-10\right)$ while the other shifts it downward $\left(t_{0}=-10.06\right)$, with the limits shown by dashed black lines. The error on the mean was obtained using 15 independent estimates of the mean in the region $3.5 \leqslant t \leqslant 10$. Here $d t=d \tau=10^{-3}, \beta=1, \Delta=1, \epsilon(t)=\kappa t$ with $\kappa=5, \omega_{c}=$ 20, $\alpha=0.01$, averaged over 1 million realizations.

the start time of the simulation. In other words, the commonly simulated spin has some "catching up" to do with respect to the true LZ spin. As we shall demonstrate below, a more appropriate initial state should recover the correct asymptotic dynamics without needing to take the particular value of $t_{0}$ into account.

For the dynamics over the period $-\infty<t \leqslant t_{0}$ the system may be approximately thermalized; this should be exact in the adiabatic limit of the LZ sweep rate $\kappa \rightarrow 0$. Hence, one possible state that we can choose at $t_{0}$ instead of the $t \rightarrow$ $-\infty$ initial LZ value of $\sigma_{z}\left(t_{0}\right)=1$ would be the equilibrium (thermalized) state associated with $\epsilon\left(t_{0}\right)$. As long as $\left|\epsilon\left(t_{0}\right)\right|$ is still much larger than the other relevant energy scales of the system, this will be a good approximation of the true LZ spin at $t_{0}$. The ESLN provides an exact way of initializing the system in this equilibrium state by the initial preparation in imaginary time, Eq. (23), and as such the full ESLN represents an improvement on SLN methods for modeling systems of this kind. Examples of the observed asymptote of the $z$ spin are shown in Fig. 8 for both initial conditions [thermalized and $\sigma_{z}\left(t_{0}\right)=1$ ] to serve as a point of comparison.

One can see that if the modified limit is shifted up or down compared to the original limit due to the finiteness of $t_{0}$, the thermalized initial condition approaches the original limit very well, regardless of the direction of the $t_{0}$ shift. Instead, it sits just above $\left\langle\sigma_{z}\right\rangle_{L Z}$ as would be expected for a finite simulation time rather than for one which runs to $t \rightarrow \infty$. This cleanly demonstrates that thermalizing the system with $\epsilon\left(t_{0}\right)$ correctly accounts for the fact that the actual system is initialized at the infinite past, and the original LZ limit is recovered. We emphasize that this has not been achieved before. 


\section{DISCUSSION AND CONCLUSIONS}

In this paper we have demonstrated a successful implementation of the extended stochastic Liouville-von Neumann equation (ESLN) computational method for obtaining realtime dynamics of the reduced density matrix of an open quantum system coupled to a harmonic bath. This method is exact and can be used for arbitrary open quantum systems at arbitrary temperature and coupling strength, at least in principle, provided that the coupling is linear in the bath coordinates. Unlike existing SLN schemes where the system is initially decoupled from the bath, in our method the combined system of the quantum system of interest and the bath are fully thermalized together, with the coupling already established. The main difference compared with SLN simulations is that the density matrix has to be initially evolved in imaginary time before the real-time propagation for each sampling trajectory.

The utility of the method has been demonstrated on two simple systems, both based on the spin-boson Hamiltonian: (i) a case where the Hamiltonian remains constant during realtime evolution, and (ii) the Landau-Zener (LZ) sweep that is a fully nonequilibrium evolution.

The first case was chosen to prove that our ESLN simulation can maintain the thermalized state at any $t>0$ and also reach it asymptotically if initialized in an arbitrary state. These simulations served as a test bed for choosing the appropriate method of generating correlated noises and establishing a computational scheme. We find that the noise generation method proposed earlier [15] represents the worst possible choice of the noises [14], in the sense that the associated dynamics are highly unstable, restricting simulation to only very short times. This presented something of a paradox, in that the correlation functions were still fully satisfied, which is the only requirement of the theory on the noises. We provide a modified scheme here, although other possibilities also exist [14]. In addition, we have also concluded that trace-preserving variants of the ESLN lead to pathological behavior and so must be discarded, leaving us with the original form of the SLN whose dynamics is not unitary. As long as an appropriate noise generation scheme is used, this turns out to be sufficient for the system to be well behaved.

Since the ESLN equations are stochastic in nature, care is required in developing an appropriate numerical scheme. We find that in our case the choice of the numerical scheme is essential if long-time simulations are needed, while for short timescales a regular Euler-Maruyama discretization is enough. For long-time simulations the higher-order schemes originating from the Stratonovich stochastic calculus, such as the Heun scheme, are advantageous.

Applications to the LZ model, in which the initial state is not thermalized, also demonstrated that our method works well even in this rather complex nonequilibrium situation. We showed that the LZ limit for a system coupled to an environment differs from that of the isolated system, but approaches it in the limit of zero environmental coupling and temperature. We have also found that in actual simulations the asymptotic limit is sensitive to the choice of the simulation time $t_{0}$ at which the system is prepared. We find that this dependence can be weakened substantially if the thermalized state corresponding to the LZ Hamiltonian at the initial time is used instead of the correct LZ initial state, demonstrating another utility of our method.

We hope that this work will stimulate further investigations of the nonequilibrium dynamics of open quantum systems by means of the ESLN method.

\section{ACKNOWLEDGMENTS}

M.A.L. and D.M. are supported by the EPSRC Centre for Doctoral Training in Cross-Disciplinary Approaches to NonEquilibrium Systems (CANES, Grant No. EP/L015854/1). Calculations in this paper were performed using the Kings College HPC cluster Gravity.

\section{APPENDIX A: GIRSANOV TRANSFORMATION}

To ensure that the normalized $\tilde{\rho}$ have the correct physical ensemble average $\rho^{p h}$, the transformation $\mathcal{W} \rightarrow \mathcal{W}^{\prime}$ must take the form

$$
\mathcal{W}^{\prime}\left[z_{1}^{\prime}, z_{2}^{\prime}\right]=\mathcal{W}\left[z_{1}, z_{2}\right] \operatorname{Tr}[\rho(t)],
$$

as can be seen by substituting $\rho(t)=\tilde{\rho}(t) \operatorname{Tr}[\rho(t)]$ into Eq. (52). Here $\operatorname{Tr}[\rho(t)]$ is easily obtained from Eq. (30) as

$$
\operatorname{Tr}[\rho(t)]=\exp \left\{\frac{i}{\hbar} \int_{0}^{t} d t^{\prime} v\left(t^{\prime}\right) \sigma\left(t^{\prime}\right)\right\} .
$$

The task is now to remove this exponential factor from the average by completing the square in $\mathcal{W}^{\prime}$ and identifying transformed noises $z^{\prime}$. Writing out Eq. (A1) explicitly, this is

$$
\begin{aligned}
\mathcal{W}^{\prime}\left[z_{1}^{\prime}, z_{2}^{\prime}\right]= & \mathcal{N} \exp \left\{-\frac{1}{2}\left[\int_{0}^{t} d t^{\prime} \int_{0}^{t} d t^{\prime \prime} z_{1}^{T}\left(t^{\prime}\right) \Sigma^{11}\left(t^{\prime}-t^{\prime \prime}\right) z_{1}\left(t^{\prime \prime}\right)+2 \int_{0}^{t} d t^{\prime} \int_{0}^{\beta \hbar} d \tau z_{1}^{T}\left(t^{\prime}\right) \Sigma^{11}\left(t^{\prime}, \tau\right) z_{2}(\tau)\right.\right. \\
& \left.\left.+\int_{0}^{\beta \hbar} d \tau \int_{0}^{\beta \hbar} d \tau^{\prime} z_{2}^{T}\left(\tau^{\prime}\right) \Sigma^{22}\left(\tau-\tau^{\prime}\right) z_{2}\left(\tau^{\prime}\right)\right]\right\} \exp \left\{\frac{i}{\hbar} \int_{0}^{t} d t^{\prime} \mathbf{S}^{T}\left(t^{\prime}\right) z_{1}\left(t^{\prime}\right)\right\},
\end{aligned}
$$

where vectors of noises $z_{1}=\left(\eta \eta^{*} v v^{*}\right)^{T}, z_{2}=\left(\mu \mu^{*}\right)^{T}$, and $z=\left(z_{1} z_{2}\right)^{T}$ and the vector $\mathbf{S}(t)=\left(\begin{array}{llll}0 & 0 & \sigma & \sigma\end{array}(t) 0\right)^{T}$ have been introduced. Note that the guide spin couples to the $v$ noise only in $\mathbf{S}(t)$. Making use of the fact that $\boldsymbol{\Sigma}$ is a symmetric matrix of time differences, and introducing its inverse via

$$
\int_{0}^{t} d t^{\prime} \boldsymbol{\Sigma}^{-1}\left(s-t^{\prime}\right) \boldsymbol{\Sigma}\left(t^{\prime}-t^{\prime \prime}\right)=\delta\left(s-t^{\prime \prime}\right),
$$


the result of completing the square in symbolic notations is

$$
-\frac{1}{2} z^{T} \boldsymbol{\Sigma} z+\frac{i}{\hbar} \mathbf{S}^{T} z=A(t)-\frac{1}{2} \boldsymbol{z}^{\prime} \boldsymbol{\Sigma} \boldsymbol{z}^{\prime},
$$

where $A(t)$ is a function independent of $z$ to be absorbed into the normalization of the physical density matrix $\mathbb{N}$, and

$$
z^{\prime}=z-\frac{i}{\hbar} \Sigma^{-1} \mathbf{S}
$$

are the transformed noises. These can be simplified by noting that the vector $\mathbf{S}$ has just one $v$ nonzero component. Hence, since $v$ is only correlated with $\eta$, only the $\eta$ noise is modified,

$$
\begin{aligned}
\eta^{\prime}(t) & =\eta(t)-\frac{i}{\hbar} \int_{0}^{t} d t^{\prime} K_{\eta \nu}\left(t-t^{\prime}\right) \sigma\left(t^{\prime}\right), \\
\nu^{\prime}(t) & =v(t), \\
\mu^{\prime}(\tau) & =\mu(\tau) .
\end{aligned}
$$

According to Eq. (52), the physical density matrix is now

$$
\boldsymbol{\rho}^{p h}(t)=\int \mathcal{D}^{2}[\eta] \mathcal{D}^{2}[v] \mathcal{D}^{2}[\mu] \mathcal{W}^{\prime}\left[\eta^{\prime}, v^{\prime}, \mu^{\prime}\right] \tilde{\rho}(t)[\eta, v, \mu],
$$

where the integrals are still performed over the original noises and their complex conjugates. Since only $\eta$ is modified, the only change of variables needed is $\eta \rightarrow \eta^{\prime}$ for which the Jacobian $J=\left|\delta \eta^{\prime} / \delta \eta\right|$ contains the elements

$$
\frac{\delta \eta^{\prime}\left(t^{\prime}\right)}{\delta \eta\left(t^{\prime \prime}\right)}=\delta\left(t^{\prime}, t^{\prime \prime}\right)-\frac{i}{\hbar} \int_{0}^{t} d s K_{\eta v}\left(t^{\prime}-s\right) \frac{\delta \sigma(s)}{\delta \eta\left(t^{\prime \prime}\right)},
$$

where $K_{\eta v}\left(t^{\prime}-s\right)$ is a known correlation function (16), independent of any particular realization of $\eta$, so does not need to be differentiated. It is also causal, requiring that $t^{\prime}>s$, as is $\sigma(s)$, so $\delta \sigma(s) / \delta \eta\left(t^{\prime \prime}\right)$ is only nonzero for $s>t^{\prime \prime}$. This bounds the integral over $s$ from $t^{\prime \prime}$ to $t^{\prime}$ which corresponds to a triangular matrix with zeros on the diagonal, and hence the integral does not contribute to the determinant $J=\left|\delta \eta^{\prime} / \delta \eta\right|$. Hence the Jacobian is simply equal to unity and applying the change of variables $\eta \rightarrow \eta^{\prime}$ completes the transformation. Since the transformed distribution has the same precision matrix $\boldsymbol{\Sigma}$ as the original distribution, the correlations for $\eta$ and $\eta^{\prime}$ have been preserved and the primes can be omitted. Replacing $\tilde{\rho}$ with $\rho$ for simplicity, we obtain the equation of motion (53) given in the main text.

\section{APPENDIX B: STRATONOVICH CORRECTION FOR THE SPIN-BOSON MODEL}

\section{Real-time propagation}

For the ESLN, it is convenient to rewrite the $2 \times 2$ density matrix as a 4-fold vector with elements $\rho_{h}^{k}$ (where $k \in[1,4]$ ) with the original matrix elements ordered as $11,12,21$, and 22. The dynamics is then split into one deterministic part and two noisy parts associated with $\eta$ and $v$,

$d \rho_{h+1}^{k}=a^{k}\left(t_{h}, \rho_{h}\right) d t+b_{\eta}^{k}\left(t_{h}, \rho_{h}\right) \eta\left(t_{h}\right) d t+b_{v}^{k}\left(t_{h}, \rho_{h}\right) v\left(t_{h}\right) d t$,

where $a^{k}\left(t_{h}, \boldsymbol{\rho}_{h}\right), b_{\eta}^{k}\left(t_{h}, \boldsymbol{\rho}_{h}\right)$, and $b_{v}^{k}\left(t_{h}, \boldsymbol{\rho}_{h}\right)$ on the right-hand side are elements of the vectors

$$
\begin{aligned}
\boldsymbol{a}\left(t_{h}, \boldsymbol{\rho}_{h}\right) & =-\frac{i}{\hbar}\left[H_{h}, \boldsymbol{\rho}_{h}\right], \\
\boldsymbol{b}_{\eta}\left(t_{h}, \boldsymbol{\rho}_{h}\right) & =\frac{i}{\hbar}\left[\sigma_{z}, \boldsymbol{\rho}_{h}\right], \\
\boldsymbol{b}_{v}\left(t_{h}, \boldsymbol{\rho}_{h}\right) & =\frac{i}{2 \hbar}\left\{\sigma_{z}, \boldsymbol{\rho}_{h}\right\},
\end{aligned}
$$

and $H_{h}=H\left(t_{h}\right)$. The noises are expressed as weighted sums of white-noise random numbers using the discretized form of Eqs. (38)-(40), where each white noise $x_{i}$ and $\bar{x}_{j}$ is expressed as $x_{j}(t) d t \rightarrow d W^{j}(t)$ and $\bar{x}_{j}(\tau) d \tau \rightarrow d \bar{W}^{j}(\tau)$, the overbar once more denoting a function of imaginary time $\tau$ and indices $j=1,2,3$ referring to specific white noises to enforce the necessary correlations. For example, the $\eta_{\eta}$ noise is expressed as

$$
\eta_{\eta}\left(t_{h}\right)=\sum_{n=-N}^{N} G_{\eta \eta}\left(t_{n}\right) d W^{1}\left(t_{h}-t_{n}\right)
$$

with $j=1$. Here we shall use the index $n$ ranging between $-N$ and $N$ to denote discretized real-time integrations with $t_{n}=n d t$, and the index $m$ between $-M$ and $M$ for the integration in imaginary time, $\tau_{m}=m d \tau$. The inverse Fourier transforms of the filtering kernels, Eqs. (44)-(46), and any other numerical prefactors can freely be absorbed into the diffusion function, giving them an additional index $n$ or $m$ associated with the appropriate sum over time. This transforms the right-hand side of Eq. (B1) into the compact form $\sum_{j} B^{k j}\left(t_{h}, \boldsymbol{\rho}_{h}\right) d W_{t}^{j}$. The rows $\mathbf{B}^{k}$ of the matrix $\boldsymbol{B}\left(t_{h}, \boldsymbol{\rho}_{h}\right)=$ $\left\{B_{h}^{k j}\right\}$ form vectors, each associated with one of the elements $\rho^{k}$ of the density vector, and are composed of the following components:

$$
\begin{aligned}
\mathbf{B}^{k}\left(t_{h}, \boldsymbol{\rho}_{h}\right)= & {\left[(\underbrace{b_{\eta}^{k} G_{\eta \eta}\left(t_{n}\right) d t}_{-N \leqslant n \leqslant N ; j \in d W^{1}})(\underbrace{b_{\eta}^{k} G_{\eta \nu}\left(t_{n}\right) d t+i b_{\nu}^{k} G_{\nu \eta}\left(t_{n}\right) d t}_{-N \leqslant n \leqslant N ; j \in d W^{2}})(\underbrace{i b_{\eta}^{k} G_{\eta \nu}\left(t_{n}\right) d t+b_{\nu}^{k} G_{\nu \eta}\left(t_{n}\right) d t}_{-N \leqslant n \leqslant N ; j \in d W^{3}})\right.} \\
& \left.(\underbrace{\left(b_{\eta}^{k} G_{\eta \mu}\left(t_{h}, \tau_{m}\right) d t\right.}_{-M \leqslant m \leqslant M ; j \in d \bar{W}^{2}})(\underbrace{i b_{\eta}^{k} G_{\eta \mu}\left(t_{h}, \tau_{m}\right) d t}_{-M \leqslant m \leqslant M ; j \in d \bar{W}^{3}})\right] .
\end{aligned}
$$

$\mathbf{B}^{k}$ contains five sets of elements, each associated with a different white noise. The index $j$ identifies the Wiener increment of the appropriate white noise, and within each set of elements the indices $n$ and $m$ run across real and imaginary times, respectively. 
For each $t_{h}$, the increments $d W^{j}$ form a $2(2 M+1)+3(2 N+1)$ long vector $d \mathbf{W}$, elements of which are ordered in the same way as inside the vector $\mathbf{B}^{k}$ above:

$$
d \mathbf{W}_{h}=\left[(\underbrace{d W^{1}\left(t_{h}-t_{n}\right)}_{-N \leqslant n \leqslant N}) \quad(\underbrace{d W^{2}\left(t_{h}-t_{n}\right)}_{-N \leqslant n \leqslant N}) \quad(\underbrace{d W^{3}\left(t_{h}-t_{n}\right)}_{-N \leqslant n \leqslant N}) \quad(\underbrace{d \bar{W}^{2}\left(\tau_{m}\right)}_{-M \leqslant m \leqslant M}) \quad(\underbrace{d \bar{W}^{3}\left(\tau_{m}\right)}_{-M \leqslant m \leqslant M})\right] .
$$

These notations enable us to refer to either of the five sets of terms in the sum $\sum_{j} B^{k j}\left(t_{h}, \rho_{h}\right) d W_{h}^{j}$ by the particular family of the noise increments, e.g., $d W^{1}$ or $d \bar{W}^{2}$, as is also indicated underneath each term in Eq. (B6). The ESLN now has the standard form of Eq. (59), and it is clear that there are many white noises appearing in this Langevin equation. This justifies the choice of using Stratonovich calculus since Itô calculus would be punitively expensive.

To transition into the Stratonovich-Heun scheme, we have to calculate the Stratonovich correction

$$
-\frac{1}{2} \sum_{l j} B^{l j}\left(t_{h}, \rho_{h}\right) \frac{\partial B^{k j}\left(t_{h}, \rho_{h}\right)}{\partial \rho_{h}^{l}}
$$

needed for the modified drift, Eq. (62), for each family of the increments $j \in d W^{1}, d W^{2}, d W^{3}, d \bar{W}^{2}$, and $d \bar{W}^{3}$. For $j \in d W^{1}$, we have $B^{k j}=b_{\eta}^{k} G_{\eta \eta}\left(t_{m}\right) d t$, and only

$$
\boldsymbol{b}_{\eta}=\frac{2 i}{\hbar}\left(\begin{array}{cc}
0 & \rho_{h}^{12} \\
-\rho_{h}^{21} & 0
\end{array}\right) \rightarrow \frac{2 i}{\hbar}\left(\begin{array}{llll}
0 & \rho_{h}^{2} & -\rho_{h}^{3} & 0
\end{array}\right)^{T}
$$

(where we have used both the $2 \times 2$ matrix and the 4 -fold vector notations) depends on the elements $\rho_{h}^{l}$ of $\rho_{h}$, so the derivatives $\partial b_{\eta}^{k} / \partial \rho_{h}^{l}$ are easily calculated forming a $4 \times 4$ matrix

$$
\left(\frac{\partial b_{\eta}^{k}}{\partial \rho_{h}^{l}}\right)=\frac{2 i}{\hbar}\left(\begin{array}{rrrr}
0 & 0 & 0 & 0 \\
0 & 1 & 0 & 0 \\
0 & 0 & -1 & 0 \\
0 & 0 & 0 & 0
\end{array}\right)
$$

with respect to indices $k, l$. Substituting these into the Stratonovich correction, we obtain the following contribution from the $d W^{1}$ terms:

$$
\begin{aligned}
& -\frac{1}{2} \sum_{l, j \in d W^{1}} B^{l j}\left(t_{h}, \boldsymbol{\rho}_{h}\right) \frac{\partial B^{k j}\left(t_{h}, \boldsymbol{\rho}_{h}\right)}{\partial \rho_{h}^{l}} \\
& \quad=2\left(\frac{d t}{\hbar}\right)^{2}\left(\begin{array}{cc}
0 & \rho_{h}^{12} \\
\rho_{h}^{21} & 0
\end{array}\right) \sum_{m=-M}^{M} G_{\eta \eta}^{2}\left(t_{m}\right),
\end{aligned}
$$

where we have returned back to the matrix notations for clarity.

Similarly, for $j \in d W^{2}$, we have $B^{k j}=i b_{\nu}^{k} G_{\nu \eta}\left(t_{m}\right) d t+$ $b_{\eta}^{k} \delta_{m 0}$, and only $\boldsymbol{b}_{v}$ and its derivative are left to calculate:

$$
\begin{aligned}
& \boldsymbol{b}_{v}=\frac{i}{\hbar}\left(\begin{array}{cc}
\rho_{h}^{11} & 0 \\
0 & -\rho_{h}^{22}
\end{array}\right) \rightarrow\left(\begin{array}{cccc}
\rho_{h}^{1} & 0 & 0 & -\rho_{h}^{4}
\end{array}\right), \\
& \left(\frac{\partial b^{k}{ }_{\nu}}{\partial \rho_{h}^{l}}\right)=\frac{i}{\hbar}\left(\begin{array}{cccc}
1 & 0 & 0 & 0 \\
0 & 0 & 0 & 0 \\
0 & 0 & 0 & 0 \\
0 & 0 & 0 & -1
\end{array}\right),
\end{aligned}
$$

and the appropriate contribution to the correction from $d W^{2}$ noises is

$$
\begin{aligned}
- & \frac{1}{2} \sum_{l, j \in d W^{2}} B^{l j}\left(t_{h}, \rho_{h}\right) \frac{\partial B^{k j}\left(t_{h}, \rho_{h}\right)}{\partial \rho_{h}^{l}} \\
= & -\frac{1}{2}\left(\frac{d t}{\hbar}\right)^{2}\left(\begin{array}{cc}
\rho_{h}^{11} & 0 \\
0 & \rho_{h}^{22}
\end{array}\right) \sum_{m=-M}^{M} G_{v \eta}^{2}\left(t_{m}\right) \\
& +\frac{2}{\hbar^{2}}\left(\begin{array}{cc}
0 & \rho_{h}^{12} \\
\rho_{h}^{21} & 0
\end{array}\right) .
\end{aligned}
$$

In the same way, the correction for $j \in d W^{3}$ is found to be identical to the correction (B13) for $j \in d W^{2}$ but with the opposite sign such that they exactly cancel.

For the Wiener increments associated with white noises in imaginary time, $j \in d \bar{W}^{2}$ and $j \in d \bar{W}^{3}$, inspection of the elements of the $\boldsymbol{B}$ matrix, Eq. (B6), reveals that the terms associated with $d \bar{W}^{3}$ are just $i$ times the terms associated with $d \bar{W}^{2}$. The Stratonovich correction for these terms will thus be identical apart from a minus sign coming from $i^{2}=-1$ in $d \bar{W}^{3}$, and they will also exactly cancel. Thus only terms from $j \in d W^{1}$ contribute to the modified drift in Eq. (62),

$$
\tilde{\boldsymbol{a}}\left(t_{h}, \boldsymbol{\rho}_{h}\right)=\boldsymbol{a}\left(t_{h}, \boldsymbol{\rho}_{h}\right)+2\left(\frac{d t}{\hbar}\right)^{2}\left(\begin{array}{cc}
0 & \rho_{h}^{12} \\
\rho_{h}^{21} & 0
\end{array}\right) \sum_{m=-M}^{M} G_{\eta \eta}^{2}\left(t_{m}\right) .
$$

It is then straightforward to convert this into the corresponding corrections for the spins $S_{x}, S_{y}, S_{z}$ and the trace $S_{\operatorname{Tr} \rho}$, Eqs. (27)-(30), yielding, respectively,

$$
\begin{gathered}
S_{x}\left(t_{h}\right)=2\left(\frac{d t}{\hbar}\right)^{2} \sum_{m=-M}^{M} G_{\eta \eta}^{2}\left(t_{m}\right) \sigma_{x}\left(t_{h}\right), \\
S_{y}\left(t_{h}\right)=2\left(\frac{d t}{\hbar}\right)^{2} \sum_{m=-M}^{M} G_{\eta \eta}^{2}\left(t_{m}\right) \sigma_{y}\left(t_{h}\right), \\
S_{z}\left(t_{h}\right)=S_{\operatorname{Tr} \rho}\left(t_{h}\right)=0, \quad \forall h .
\end{gathered}
$$

\section{Imaginary-time propagation}

It is straightforward to repeat the same procedure for thermalization, Eq. (23),

$$
\bar{\rho}_{h+1}^{k}=\bar{\rho}_{h}^{k}-H_{0} \bar{\rho}_{h}^{k} d \tau+\sigma_{z} \bar{\rho}_{h}^{k} \mu\left(\tau_{h}\right) d \tau,
$$

where $H_{0}$ is the initial Hamiltonian at the beginning of the real-time evolution, and $h$ is now an index associated with the imaginary time $\tau_{h}=h d \tau$. There is no additional complexity here as compared to the real-time evolution, so for expedience the result for the modified drift is simply stated

$$
\tilde{\boldsymbol{a}}\left(\tau_{h}, \overline{\boldsymbol{\rho}}_{h}\right)=-\left[H_{0}+\frac{1}{2}\left(\frac{d \tau}{\hbar}\right)^{2} \sum_{n=-N}^{N} G_{\mu \mu}^{2}\left(\tau_{n}\right)\right] \overline{\boldsymbol{\rho}}_{h} .
$$


[1] P. W. Shor, Scheme for reducing decoherence in quantum computer memory, Phys. Rev. A 52, R2493 (1995).

[2] U. Weiss, Quantum Dissipative Systems (World Scientific, 2012).

[3] R. P. Feynman and F. L. Vernon, Jr., The theory of a general quantum system interacting with a linear dissipative system, Ann. Phys. 281, 547 (2000).

[4] Y. Yan, F. Yang, Y. Liu, and J. Shao, Hierarchical approach based on stochastic decoupling to dissipative systems, Chem. Phys. Lett. 395, 216 (2004).

[5] D. Suess, A. Eisfeld, and W. T. Strunz, Hierarchy of Stochastic Pure States for Open Quantum System Dynamics, Phys. Rev. Lett. 113, 150403 (2014).

[6] L. Diósi, N. Gisin, and W. T. Strunz, Non-Markovian quantum state diffusion, Phys. Rev. A 58, 1699 (1998).

[7] J. T. Stockburger and H. Grabert, Non-Markovian quantum state diffusion, Chem. Phys. 268, 249 (2001).

[8] J. T. Stockburger and H. Grabert, Exact C-Number Representation of Non-Markovian Quantum Dissipation, Phys. Rev. Lett. 88, 170407 (2002).

[9] J. T. Stockburger, Simulating spin-boson dynamics with stochastic Liouville-von Neumann equations, Chem. Phys. 296, 159 (2004).

[10] G. M. G. McCaul, C. D. Lorenz, and L. Kantorovich, Partitionfree approach to open quantum systems in harmonic environments: An exact stochastic Liouville equation, Phys. Rev. B 95, 125124 (2017).

[11] P. P. Orth, A. Imambekov, and K. Le Hur, Nonperturbative stochastic method for driven spin-boson model, Phys. Rev. B 87, 014305 (2013).

[12] N. Makri and D. E. Makarov, Tensor propagator for iterative quantum time evolution of reduced density matrices. I. Theory, J. Chem. Phys. 102, 4600 (1995).

[13] H. Grabert, P. Schramm, and G. Ingold, Quantum Brownian motion: The functional integral approach, Phys. Rep. 168, 115 (1988).

[14] D. Matos, M. A. Lane, I. Ford, and L. Kantorovich, arXiv:2006.01863 [Phys. Rev. B (to be published)].

[15] G. M. G. McCaul, C. D. Lorenz, and L. Kantorovich, Driving spin-boson models from equilibrium using exact quantum dynamics, Phys. Rev. B 97, 224310 (2018).

[16] H. Imai, Y. Ohtsuki, and H. Kono, Application of stochastic Liouville-von Neumann equation to electronic energy transfer in FMO complex, Chem. Phys. 446, 134 (2015).

[17] K. Schmitz and J. T. Stockburger, A variance reduction technique for the stochastic Liouville-von Neumann equation, Eur. Phys. J.: Spec. Top. 227, 1929 (2019).

[18] L. Duan and G. Guo, Reducing decoherence in quantumcomputer memory with all quantum bits coupling to the same environment, Phys. Rev. A 57, 737 (1998).

[19] T. A. Costi and R. H. McKenzie, Entanglement between a qubit and the environment in the spin-boson model, Phys. Rev. A 68 , 034301 (2003).

[20] C. H. van der Wal, F. K. Wilhelm, C. J. P. M. Harmans, and J. E. Mooij, Engineering decoherence in Josephson persistent-current qubits, Eur. Phys. J. B 31, 111 (2003).

[21] A. Kopp and K. Le Hur, Universal and Measurable Entanglement Entropy in the Spin-Boson Model, Phys. Rev. Lett. 98, 220401 (2007).
[22] W. Cui, Z. Xi, and Y. Pan, Non-Markovian entanglement dynamics between two coupled qubits in the same environment, J. Phys. A: Math. Theor. 42, 155303 (2009).

[23] Y. Makhlin, G. Schön, and A. Shnirman, Quantum-state engineering with Josephson-junction devices, Rev. Mod. Phys. 73, 357 (2001).

[24] J. Liu, L. Fu, B. Ou, S. Chen, D. Choi, B. Wu, and Q. Niu, Theory of nonlinear Landau-Zener tunneling, Phys. Rev. A 66, 023404 (2002).

[25] D. Valenti, C. Guarcello, and B. Spagnolo, Switching times in long-overlap Josephson junctions subject to thermal fluctuations and non-Gaussian noise sources, Phys. Rev. B 89, 214510 (2014).

[26] P. P. Orth, D. Roosen, W. Hofstetter, and K. Le Hur, Dynamics, synchronization, and quantum phase transitions of two dissipative spins, Phys. Rev. B 82, 144423 (2010).

[27] P. P. Orth, I. Stanic, and K. Le Hur, Dissipative quantum Ising model in a cold-atom spin-boson mixture, Phys. Rev. A 77, 051601 (2008).

[28] D. M. Berns, M. S. Rudner, S. O. Valenzuela, K. K. Berggren, W. D. Oliver, L. S. Levitov, and T. P. Orlando, Amplitude spectroscopy of a solid-state artificial atom, Nature (London) 455, 51 (2008).

[29] I. V. Girsanov, On transforming a certain class of stochastic processes by absolutely continuous substitution of measures, Theory Probab. Appl. 5, 285 (1960).

[30] A. O. Caldeira and A. J. Leggett, Path integral approach to quantum Brownian motion, Phys. A (Amsterdam) 121, 587 (1983).

[31] H. P. Breuer and J. Piilo, Stochastic jump processes for non-Markovian quantum dynamics, Europhys. Lett. 85, 50004 (2009).

[32] Z. Ruan and R. Baer, Unravelling open-system quantum dynamics of non-interacting fermions, Mol. Phys. 116, 2490 (2018).

[33] M. Moodley and F. Petruccione, Stochastic wave-function unraveling of the generalized Lindblad master equation, Phys. Rev. A 79, 042103 (2009).

[34] J. Hubbard, Calculation of Partition Functions, Phys. Rev. Lett. 3, 77 (1959).

[35] H.-P. Breuer, F. Petruccione et al., The Theory of Open Quantum Systems (Oxford University Press on Demand, 2002).

[36] C. Zener, Non-adiabatic crossing of energy levels, Proc. R. Soc. London A 137, 696 (1932).

[37] C. Wittig, The Landau-Zener formula, J. Phys. Chem. B 109, 8428 (2005).

[38] A. G. Rojo, Matrix exponential solution of the Landau-Zener problem, arXiv:1004.2914.

[39] K. Saito, M. Wubs, S. Kohler, Y. Kayanuma, and P. Hänggi, Dissipative Landau-Zener transitions of a qubit: Bath-specific and universal behavior, Phys. Rev. B 75, 214308 (2007).

[40] P. Nalbach and M. Thorwart, Landau-Zener Transitions in a Dissipative Environment: Numerically Exact Results, Phys. Rev. Lett. 103, 220401 (2009).

[41] M. Wubs, K. Saito, S. Kohler, P. Hänggi, and Y. Kayanuma, Gauging a Quantum Heat Bath with Dissipative Landau-Zener Transitions, Phys. Rev. Lett. 97, 200404 (2006).

[42] J. Dávila, J. Fernández Bonder, J. D. Rossi, P. Groisman, and M. Sued, Numerical analysis of stochastic differential equations with explosions, Stoch. Anal. Appl. 23, 809 (2005). 
[43] D. J. Higham, An algorithmic introduction to numerical simulation of stochastic differential equations, SIAM Rev. 43, 525 (2001).

[44] G. C. Ghirardi, P. Pearle, and A. Rimini, Markov processes in Hilbert space and continuous spontaneous localization of systems of identical particles, Phys. Rev. A 42, 78 (1990).

[45] D. Gatarek and N. Gisin, Continuous quantum jumps and infinite-dimensional stochastic equations, J. Math. Phys. 32, 2152 (1991).

[46] C. Gardiner, Stochastic Methods (Springer, Berlin, 2009).

[47] P. E. Kloeden, E. Platen, and H. Schurz, Numerical Solution of SDE through Computer Experiments (Springer Science \& Business Media, 2012).

[48] G. F. Lawler, Introduction to Stochastic Processes (Chapman and Hall, 2018).

[49] W. T. Strunz, L. Diósi, and N. Gisin, Open System Dynamics with Non-Markovian Quantum Trajectories, Phys. Rev. Lett. 82, 1801 (1999).

[50] Y. Tanimura, Stochastic Liouville, Langevin, FokkerPlanck, and master equation approaches to quantum dissipative systems, J. Phys. Soc. Jpn. 75, 082001 (2006).

[51] H. Risken, Fokker-Planck equation, The Fokker-Planck Equation (Springer, 1996).

[52] P. E. Kloeden and E. Platen, Numerical Solution of Stochastic Differential Equations, 1 ed. (Springer-Verlag Berlin, Heidelberg, 2013), Vol. 1.

[53] E. Platen, An introduction to numerical methods for stochastic differential equations, Acta Numerica 8, 197 (1999).

[54] C. W. Gardiner et al., Handbook of Stochastic Methods (Springer, Berlin, 1985).

[55] S. Särkkä and A. Solin, Applied Stochastic Differential Equations (Cambridge University Press, 2019).

[56] E. Tzitzili, Numerical approximation of Stratonovich SDEs and SPDEs, Ph.D. thesis, Department of Mathematics, Heriot-Watt University, 2015.

[57] W. Rümelin, Numerical treatment of stochastic differential equations, SIAM J. Numer. Anal. 19, 604 (1982).

[58] A. J. Leggett, S. Chakravarty, A. T. Dorsey, M. P. A. Fisher, A. Garg, and W. Zwerger, Dynamics of the dissipative two-state system, Rev. Mod. Phys. 59, 1 (1987). 